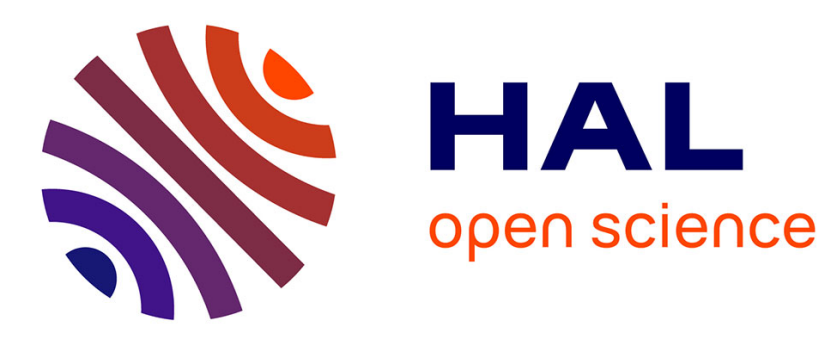

\title{
Scalable Interactive Dynamic Graph Clustering on Multicore CPUs
}

\author{
Son Mai, Sihem Amer-Yahia, Ira Assent, Mathias Skovgaard Birk, Martin \\ Storgaard Dieu, Jon Jacobsen, Jesper Kristensen
}

\section{To cite this version:}

Son Mai, Sihem Amer-Yahia, Ira Assent, Mathias Skovgaard Birk, Martin Storgaard Dieu, et al.. Scalable Interactive Dynamic Graph Clustering on Multicore CPUs. IEEE Transactions on Knowledge and Data Engineering, 2019, 31 (7), pp.1239-1252. 10.1109/TKDE.2018.2828086 . hal-02972587

\section{HAL Id: hal-02972587 \\ https://hal.science/hal-02972587}

Submitted on 12 Nov 2020

HAL is a multi-disciplinary open access archive for the deposit and dissemination of scientific research documents, whether they are published or not. The documents may come from teaching and research institutions in France or abroad, or from public or private research centers.
L'archive ouverte pluridisciplinaire HAL, est destinée au dépôt et à la diffusion de documents scientifiques de niveau recherche, publiés ou non, émanant des établissements d'enseignement et de recherche français ou étrangers, des laboratoires publics ou privés. 


\title{
Scalable Interactive Dynamic Graph Clustering on Multicore CPUs
}

\author{
Son T. Mai, Sihem Amer-Yahia \\ CNRS, University of Grenoble Alpes, France \\ Email: \{mtson, sihem.amer-yahia\}@univ-grenoble-alpes.fr \\ Ira Assent, Mathias Birk, Martin Storgaard Dieu, Jon Jacobsen, Jesper Kristensen \\ Department of Computer Science, Aarhus University, Denmark \\ Email: ira@cs.au.dk
}

\begin{abstract}
The structural graph clustering algorithm SCAN is a fundamental technique for managing and analyzing graph data. However, its high runtime remains a computational bottleneck, which limits its applicability. In this paper, we propose a novel interactive approach for tackling this problem on multicore CPUs. Our algorithm, called anySCAN, iteratively processes vertices in blocks. The acquired results are merged into an underlying cluster structure consisting of the so-called super-nodes for building clusters. During its runtime, anySCAN can be suspended for examining intermediate results and resumed for finding better results at arbitrary time points, making it an anytime algorithm which is capable of handling very large graphs in an interactive way and under arbitrary time constraints. Moreover, its block processing scheme allows the design of a scalable parallel algorithm on shared memory architectures such as multicore CPUs for speeding up the algorithm further at each iteration. Consequently, anySCAN uniquely is a both interactive and work-efficient parallel algorithm. We further introduce danySCAN an efficient bulk update scheme for anySCAN on dynamic graphs in which the clusters are updated in bulks and in a parallel interactive scheme. Experiments are conducted on very large real graph datasets for demonstrating the performance of anySCAN. They show its ability to acquire very good approximate results early, leading to orders of magnitude speedup compared to SCAN and its variants. Moreover, it scales very well with the number of threads when dealing with both static and dynamic graphs.
\end{abstract}

Index Terms-Structural graph clustering, SCAN, anytime clustering, parallel algorithm, multicore CPUs, dynamic graphs.

\section{INTRODUCTION}

Given a graph $G=(V, E)$, where $V$ is a set of vertices and $E$ a set of edges, graph clustering algorithms group vertices in $V$ so that those in the same group are highly connected and there are few connections among different groups. They have many applications, e.g., finding communities of people in social networks or detecting hidden structures in graphs. During the last decades, many techniques have been introduced such as modularity-based methods [1] and graph partitioning [2]. Among these techniques, the structural graph clustering algorithm SCAN [3] is not only able to discover clusters but also hubs connecting several clusters and outliers. SCAN, however, requires evaluating all $O(|E|)$ structural similarities for all pairs of adjacent vertices. For very large graphs, these overheads obviously are a computational bottleneck that limits applicability. Enhancing the performance of SCAN is thus an important task and is currently attracting considerable research efforts, e.g., [4][9]. However, many challenges still remain. For example, how can we produce clusters under arbitrary time constraints? How can we provide user interaction during the clustering process? How can we design a parallel algorithm that scales well with multiple threads while still efficient enough compared to state-of-the-art sequential techniques under single thread usage? Or, how can we efficiently deal with changes in dynamic graphs?

Contributions. In this paper, we focus on the problem of speeding up SCAN for very large graphs and deal with all questions above. Our algorithm, called anytime SCAN (anySCAN), has some unique properties described below.

First, anySCAN, as an anytime technique, quickly produces an approximate result and then iteratively refines it during its execution. Thus, while it is running, users can suspend it for examining intermediate results and resume it again at any time, until a satisfactory result is reached. Obviously, this interactive scheme of anySCAN is very useful for coping with very large graphs under arbitrary limited time constraints. Though anytime algorithms have been widely employed for time consuming problems in many fields, e.g. [10]-[12], no anytime variant of SCAN exists.

Second, anySCAN is the first parallel extension of SCAN specifically designed for shared memory architectures such as multicore CPUs. By maintaining an underlying cluster structure consisting of the so-called super-nodes and processing vertices in blocks to connect these super-nodes to form clusters, anySCAN significantly reduces synchronization among threads, thus making it a scalable parallel algorithm. Combined with its anytime property, anySCAN is an unique technique that can exploit multiple threads for approximating the results of SCAN as well as produce the exact results of the algorithm SCAN faster.

Third, anySCAN is a work-efficient anytime and parallel method. Concretely, an anytime version of an algorithm usually ends up being slower if run to the end than the original algorithm due to additional cost for maintaining 
the anytime properties. Similarly, a parallel algorithm aims to ensure high throughput to better utilize all its threads. Typically high throughput comes at the cost of increased overall workload, making it slower on a single thread than sequential techniques. In contrast to these techniques, by examining the current cluster structure at each iteration and calculating the structural similarity only when it is necessary, anySCAN reduces redundant calculations and is thus a work-efficient method, i.e., its final cumulative runtimes are much faster than SCAN even on a single thread.

Extensions. In our previous work [13], we only consider static graphs. However, many real-world graphs such as social networks (e.g., Facebook or Twitter), communication networks, or biological networks are subject to frequent changes. For example, an edge is added or removed when two persons are connected or disconnected in Facebook, leading to changes in communities. These changes are even more volatile when considering actions taken in the network (such as sharing particular types of content) to identify communities. In these cases, the graph clustering should be updated immeditately to reflect these changes, instead of reclustering from scratch.

When graphs evolve over time, all existing techniques for SCAN update clusters in a batch mode for each change [8], which may not be efficient when the number of changes is large due to redundant calculations. In contrast, our algorithm, called danySCAN, processes changes in a bulk mode for multiple updates at once. It thereby achieves performance improvements as well as admits an interactive parallel processing scheme for updating clusters. To the best of our knowledge, danySCAN is the first dynamic clustering algorithm with interactive parallel bulk update for SCAN.

Experiments are conducted on very large graphs with up to 1.3 billion edges to demonstrate the performance of our algorithms. They are an order of magnitude faster than SCAN and its variants. Moreover, in its parallel mode, they scale very well with the number of threads on both static and dynamic graphs.

\section{SOME BASIC Notions}

The algorithm SCAN. SCAN [3] is originally designed for clustering undirected and unweighted graphs. Here, we study weighted graphs, which are more general and have wider applicability. Thus, we first extend the notion of SCAN to work with weighted graphs.

We are given an undirected and weighted graph $G=$ $(V, E, W)$, where $V, E, W$ are sets of vertices, edges, and their weights of $G$, respectively. Let $N_{p}$ be the set of adjacency vertices of a vertex $p$, and $w_{p q} \in W$ be the weight of the edge $(p, q)$. We extend the unweighted structural similarity notion of SCAN into a weighted one as follows.

Definition 1. The weighted structural similarity between two vertices $p$ and $q$ is defined as:

$$
\sigma(p, q)=\left(\sum_{r \in N_{p} \cap N_{q}} w_{p r} \cdot w_{q r}\right) / \sqrt{\left(\sum_{r \in N_{p}} w_{p r}^{2}\right) \cdot\left(\sum_{r \in N_{q}} w_{q r}^{2}\right)}
$$

Generally, $\sigma(p, q)$ indicates how strong the two vertices influence each other through their shared neighbors. The structural similarity of SCAN is a special case of Definition

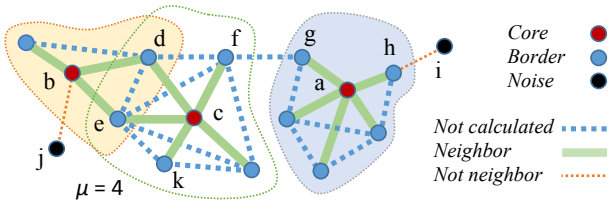

Fig. 1. Basic idea of anySCAN

1 where all the edge weights are 1. Similar to [3], $\sigma(p, q)$ can be calculated in $O\left(\left|N_{p}\right|+\left|N_{q}\right|\right)$ time following the sortmerge join style [5] or $O\left(\min \left(\left|N_{p}\right|,\left|N_{q}\right|\right)\right)$ if a hash structure is employed [5] since the length $l_{p}=\sum_{r \in N_{p}} w_{p r}^{2}$ of a vertex $p$ is fixed and can be easily calculated in a preprocessing step in $O\left(\left|N_{p}\right|\right)$ time. Given the two parameters $\mu \in \mathbb{N}^{+}$and $\epsilon \in \mathbb{R}^{*}$, the cluster notions of SCAN can be extended by using the weighted structural similarity in Definition 1.

Definition 2. The structural neighborhood of a vertex $p\left(N_{p}^{\epsilon}\right)$ is defined as $N_{p}^{\epsilon}=\left\{q \mid q \in N_{p} \wedge \sigma(p, q) \geq \epsilon\right\}$.

Definition 3. A vertex $p$ is called a core vertex, denoted as core $(p)$, if $\left|N_{p}^{\epsilon}\right| \geq \mu$. If $p$ has less than $\mu$ neighbors but one of its neighbors is a core vertex, then it is called a border (denoted as border $(p)$ ). Otherwise it is called noise (or outlier) (denoted as noise $(p)$ ).

Definition 4. A vertex $p$ is directly density-reached from $q$, denoted as $p \triangleleft q$, if $\operatorname{core}(q)$ and $p \in N_{q}^{\epsilon}$. Two vertices $p$ and $q$ are density-connected, denoted as $p \bowtie q$, if there exists a chain of vertices $x$ so that $p \triangleleft x_{1} \cdots \triangleleft x_{i} \triangleright \cdots \triangleright x_{n} \triangleright q$.

Definition 5. A cluster in SCAN is defined as a maximal set of vertices that are density-connected from each other.

SCAN builds clusters by randomly starting from an unprocessed core vertex $p$, finding its neighbors $q$, and expanding clusters by examining $q$ 's neighbors until all vertices are processed. Its time complexity is $O\left(\min \left(\left|N_{p}\right|,\left|N_{q}\right|\right) \cdot|E|\right)$ and it is worst-case optimal [5].

Anytime algorithms. Anytime algorithms are widely used to cope with time consuming tasks in many fields, e.g., [10], [12], [14]-[16]. In contrast to batch algorithms, anytime ones can be interrupted to provide a best-so-far result and then resumed to produce better results at any time, thus allowing interactions with users during execution.

\section{The Algorithm Anytime SCAN}

Figure 1 illustrates the basic idea of anySCAN. It processes vertices such that the cluster structure quickly emerges and is refined without naively checking all vertices. Assume that with the first five neighborhood checks on $a, b, c, i$, and $j$, we know that $a, b$, and $c$ are core vertices, and $i$ and $j$ are noise. Following Definition 4 and 5, all other vertices now belong to at least one cluster, e.g., $g$ and $h$ must be in the same cluster with $a$. AnySCAN then looks for true clusters using this information as a guideline. If it chooses $d$ and discovers that core $(d)$, then $b, c$ and all their neighbors $\left(N_{b}^{\epsilon}\right.$ and $N_{c}^{\epsilon}$ ) must belong to the same cluster. Next, it examines $f$ and sees that $f$ is not a core, then $N_{a}^{\epsilon}$ and $N_{c}^{\epsilon}$ will surely belong to different clusters. It can then safely stop the algorithm and have the same clustering result as SCAN without further examining the rest of the vertices, since the clusters will not change anymore regardless of additional neighborhood checks. This saves many structural similarity 


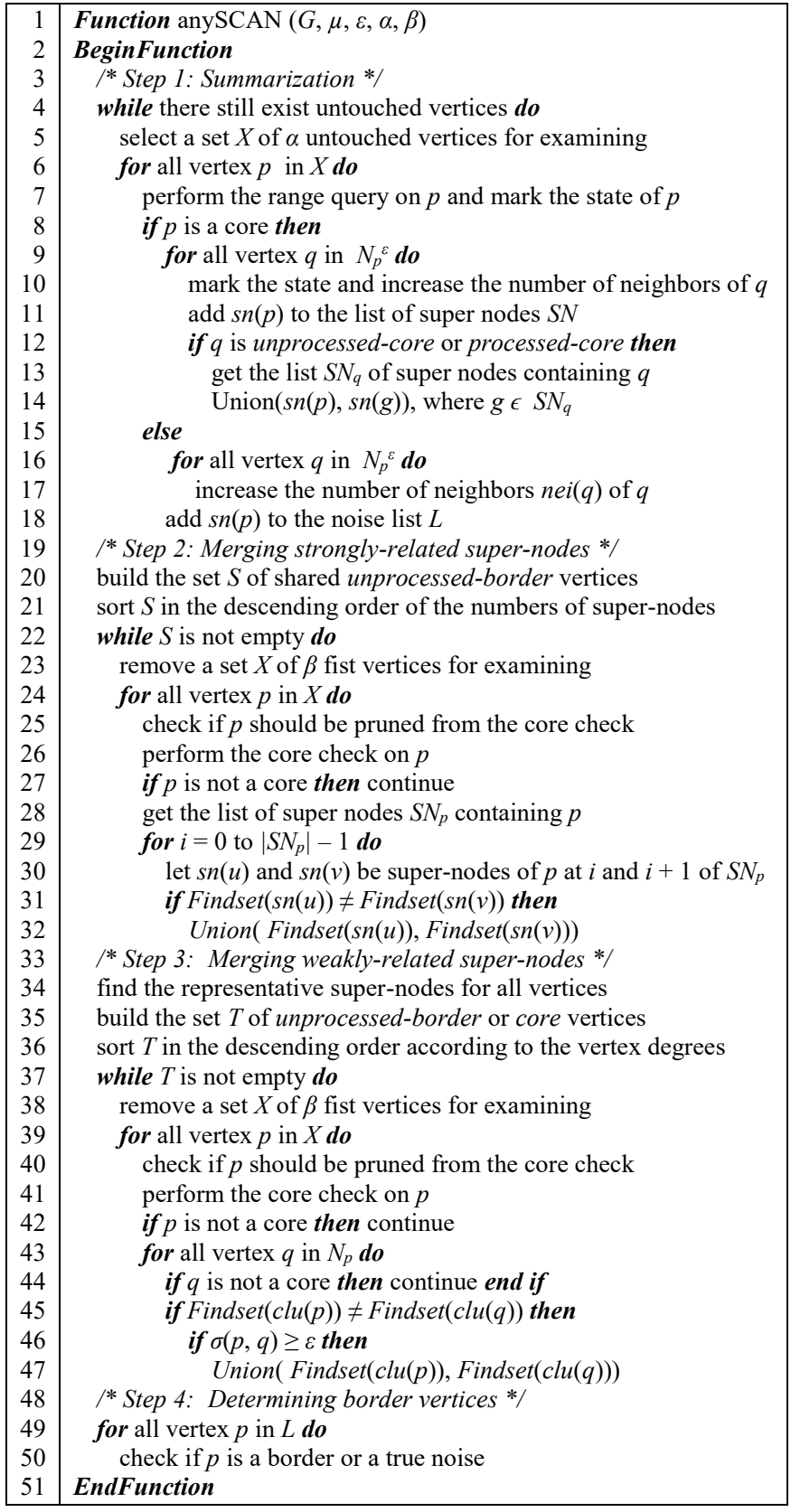

Fig. 2. Pseudocode for anySCAN

calculations and reduces runtime. Moreover, anySCAN can be interrupted after any neighborhood checks for producing approximate results of SCAN. Besides that, if it processes a block of vertices (with sizes $\alpha$ and $\beta$ ) each time instead of a single vertex like SCAN and its variants [3]-[5], each neighborhood query can be handled by a thread independently before being used to produce clusters, thus opening a way for designing a scalable parallel technique on shared memory architectures such as multicore processors.

\subsection{Anytime SCAN}

Concretely, anySCAN is built upon the concepts: summarization, selection, merging, and block processing which are summarized in Figure 2.

Step 1: Summarization. Step 1 (Line 3-18) summarizes vertices into homogeneous groups called super-nodes, which will be exploited to build clusters quickly in the next steps.

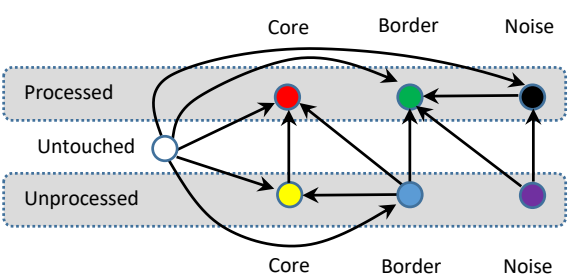

Fig. 3. The state transition schema for vertices

Assume that all vertices have untouched states in the beginning. We repeatedly and randomly choose an untouched vertice $p$ for examining until there are no more untouched ones. If noise (p) (Line 15-18), we mark it as processed-noise and store $N_{p}^{\epsilon}$ in a noise list $L$ for a post processing step. If $\operatorname{core}(p)$ (Line 8-14), $N_{p}^{\epsilon}$ is summarized in a super-node with $p$ as a representative (denoted as $s n(p)$ ) and stored in the super-node list $S N$ for further processing. We update the states of $p$ to processed-core and its neighbors $q$ to processedborder (if $q$ was noise), or unprocessed-border (if $q$ is not examined), or unprocessed-core (if $q$ is untouched and is known to have more than $\mu$ neighbors) ${ }^{1}$ following the vertex transition state schema in Figure 3 described below.

Figure 3 summarizes the state transition for all vertices during the execution of anySCAN. For example, if $\left|N_{p}\right|<\mu$, then we know that $p$ is surely not a core without having to examine its neighbors. Thus state $(p)$ is unprocessed-noise. If we know that $p$ is a neighbor of a core vertex $q$, then $p$ will be a border of a cluster. Thus its state is changed to processedborder. If none of its neighbors is core, $p$ is assigned processednoise state. If an unprocessed-border object $p$ has more than $\mu$ neighbors, it is surely a core and is changed to processed-core. Otherwise if it has fewer than $\mu$ neighbors, it is changed to processed-border since it already belongs to a cluster. If an unprocessed-border vertex $p$ is not examined but $p$ has more than $\mu$ neighbors then it is surely a core and is assigned unprocessed-core state. A border vertex will never become a core. A core vertex will not change to a border or a noise one. A processed vertex will not change to unprocessed.

Lemma 1. The state of each vertex in anySCAN changes according to the transition schema in Figure 3.

Lemma 1 can be verified through Definition 3 and 4 of SCAN. As demonstrated in Figure 1, after the summarization step, we have three super-nodes $s n(a), s n(b)$, and $s n(c)$. Noise list $L$ contains two vertices $i$ and $j$. All other vertices are not examined and marked as unprocessed-border.

Lemma 2. All objects inside a super-node $s n(p)$ belong to the same cluster.

Lemma 2 is directly inferred from Definition 4. Following it, we only need to label all the super-nodes instead of labeling all vertices like SCAN. Since the number of supernodes is much smaller than the number of vertices, the label propagation time is reduced. To do so, each supernode is initially placed in a single cluster. If we discover that $\operatorname{sn}(p)$ and $\operatorname{sn}(q)$ must belong to the same cluster, we merge them (Line 12-14). Here, a Disjoint-set data structure [17] is employed to keep track of the labels of super-nodes. It supports two operations including (1) Findset: for finding

1. To do so, we additionally store for each object $q$ the number of neighbors it currently has, denoted as nei $(q)$. 
which subset a particular super-node is in and (2) Union: for merging two subsets of super-nodes into a single subset.

At any time (e.g., after each iteration of anySCAN), the intermediate clustering result of anySCAN can be acquired by labeling a vertex according to the label of its super-node.

Step 2: Merging strongly-related super-nodes. In Step 2 (Line 19-32), anySCAN merges super-nodes to form clusters starting with ones that are more likely to be in the same cluster, e.g., vertices $d$ and $e$ in Figure 1 .

Definition 6. Two super-nodes $\operatorname{sn}(p)$ and $\operatorname{sn}(q)$ are called strongly-related to each other if they share some vertices, i.e., $\operatorname{sn}(p) \cap \operatorname{sn}(q) \neq \emptyset$.

Intuitively, if $s n(p)$ and $s n(q)$ are strongly-related, they have a high chance to be in the same cluster. Thus, in this step, we will examine all pairs of strongly-related supernodes to see if they should be merged together.

Lemma 3. If there exists a core vertex $u \in \operatorname{sn}(p) \cap \operatorname{sn}(q)$ (either in processed or unprocessed state), $s n(p)$ and $\operatorname{sn}(q)$ belong to the same cluster.

Proof 1. Let $a$ and $b$ be two arbitrary vertices in $\operatorname{sn}(p)$ and $s n(q)$, respectively. We have $a \triangleleft p$ and $q \triangleright b$ (Definition 4). Since core $(u)$ and $u \in N_{p}^{\epsilon} \cap N_{q}^{\epsilon}, p \triangleleft u$ and $u \triangleright q, a$ and $b$ are density-connected according to Definition 4.

Lemma 3 states that if two super-nodes share a core vertex, they are merged. We first collect a set $S$ of all unprocessed-border vertices that belong to at least two supernodes (Line 20), e.g., vertices $d$ and $e$ in Figure 1. The rest can be safely ignored since they do not help to determine the new connection of two super-nodes (cores has been processed in Step 1). We sort all vertices in $S$ in descending order of the numbers of super-nodes they belong to (Line 21). Then, each vertex is extracted and processed to merge super-nodes until $S$ is empty.

For each vertex $p$, if all its super-nodes already belong to the same cluster, we do not need to examine $p$ anymore since it will not lead to any change in the result (Line 25). Otherwise, if $p$ is an unprocessed-border vertex, we need to check if it is a core (Line 26). To do so, we explore its adjacency vertices $q \in N_{p}$ until we know that $p$ is a core, i.e., it has more than $\mu$ neighbors, instead of calculating all structural similarities around $p$. This helps to reduce the runtime. If $p$ is a core, we set its state to unprocessed-core. Otherwise, it is changed to processed-border vertex following the transition schema in Figure 3. Note that, we still mark the states for neighbors of $p$ as in Step 1 and 3. Now, if $p$ is a core, all of its super-nodes $g \in S N_{p}$ belong to the same cluster due to Lemma 3. Hence, anySCAN calls at most $\left|S N_{p}\right|-1$ Union operations for merging them together where $S N_{p}$ is the set of super-nodes that contains $p$ (Line 29-32). Here sorting (Line 21) can help to reduce the number of core checks since many super-nodes will be merged earlier.

Figure 1 shows an example of Step 2. Only $d$ and $e$ need to be examined. We first examine $d$ and see that it is a core. Then $s n(b)$ and $s n(c)$ are merged into one cluster. Now, $e$ can be ignored since all of its super-nodes have the same label. However, to finally conclude that the results are completely identical to SCAN, we need to check whether $\operatorname{sn}(a)$ and $\operatorname{sn}(c)$ should be merged in Step 3.
Step 3: Merging weakly-related super-nodes. Step 3 (Line 33-47) verifies connections that cannot be discovered in Step 2 , e.g., $\operatorname{sn}(a)$ and $\operatorname{sn}(c)$.

Definition 7. Two super-nodes $s n(p)$ and $s n(q)$ are weaklyrelated if there exist two vertices $u \in s n(p)$ and $v \in s n(q)$ so that $u \in N_{v}$ and $v \in N_{u} v$, i.e., $u$ and $v$ are adjacent.

Lemma 4. If there exist two core vertices $u \in \operatorname{sn}(p)$ and $v \in \operatorname{sn}(q)$ so that $u$ and $v$ are adjacent (i.e., $(u, v) \in E$ ) and $\sigma(u, v) \geq \epsilon$ then two super-nodes $\operatorname{sn}(p)$ and $\operatorname{sn}(q)$ belong to the same cluster.

Proof 2. Let $a$ and $b$ be two arbitrary vertices in $\operatorname{sn}(p)$ and $\operatorname{sn}(q)$, respectively. We have $a \triangleleft p$ and $q \triangleright b$ (Definition 4). Since core $(u)$ and $\operatorname{core}(v)$ and $\sigma(u, v) \geq \epsilon$, we have $u \bowtie v, p \triangleleft u$, and $v \triangleright q$ (Definition 4). Thus, $a$ and $b$ are density-connected (Definition 4).

Identifying the connection between weakly-connected super-nodes is much more difficult than for the stronglyconnected case. Simply examining every pair of super-nodes $s n(p)$ and $s n(q)$, identifying the edge $(p, q)$ that potentially connects them, and then checking their connection is clearly expensive. Therefore, we start with a set $T$ of all unprocessed-border, unprocessed-core, or processed-core vertices (all processed-border and noise can be safely ignored due to Lemma 4) (Line 35). We first sort $T$ in descending order of vertex degrees (Line 36). Each vertex is extracted and processed until $T$ is empty. The intuition behind this is that the higher the degree of a vertex $p$, the more likely it connects more super-nodes. Thus, examining it earlier helps to save core checks for the next ones.

For each vertex $p$, we scan all of its adjacent vertices $q$ to see if they belong to the same cluster. If so, we can safely skip $p$ since examining $p$ will not lead to any change in the clustering result (Line 40). Otherwise, $p$ may belong to an edge that connects two weakly-related super-nodes, and thus needs to be examined. If $p$ is an unprocessed-border vertex, we need to check if it is a core one like in Step 2 (Line 41). If $p$ is a core, we set its state to unprocessed-core. Otherwise, it is changed to processed-border following Figure 3. If $p$ is a core, we again scan through its adjacent vertices $q \in N_{p}$. If $q$ is not a core, we can skip it. Otherwise, if $p$ and $q$ belong to different clusters (Line 45), we calculate their structural similarity $\sigma(p, q)$. If $\sigma(p, q) \geq \epsilon$, we merge $\operatorname{sn}(u)$ and $\operatorname{sn}(v)$ by calling the Union operation, where $\operatorname{sn}(u)=\operatorname{clu}(p)$ and $\operatorname{sn}(v)=\operatorname{clu}(q)$ (Line 46-47), where $\operatorname{clu}(p)$ is the cluster that contains $p$. Note that after Step 2, all unprocessed-border or unprocessed-core vertices only belong to one cluster.

In Figure 1, when we examine $k$, all of its adjacent vertices belong to the same cluster. Thus, $k$ is skipped. However, $g$ and adjacent vertex $f$ belong to different clusters. Thus, $g$ has to be checked. In this case, $g$ is not a core. Thus, $f$ and $g$ belong to different clusters.

Step 4: Determining border vertices. Recall that in Step 1 , all noise vertices are placed in a noise list $L$. However, some of them may be border vertices if they are connected to core ones. In Step 4 (Line 49-50), we detect this case. For all vertices $p \in L$, if $\operatorname{state}(p)$ is processed-noise, we examine all $q \in N_{p}^{\epsilon}$. If $q$ is a core either processed or unprocessed, $p$ is a border of $c l u(p)$. If $q$ is unprocessed-border, we need to check if $q$ is a core and $\sigma(p, q) \geq \epsilon$. If so, $p$ is a border of 
$c l u(p)$. If $p$ is unprocessed-noise, we examine all $q \in N_{p}$. If $q$ is processed-core or unprocessed-core and $\sigma(p, q) \geq \epsilon$ then $p$ belongs to the same cluster as $q$. If $q$ is unprocessed-border, we check if $q$ is a core and $\sigma(p, q) \geq \epsilon$. If so, $p$ and $q$ belong to the same cluster. By checking noise at the end, we can exploit previous results such as core vertices to save more on similarity calculation, thus enhancing performance.

\subsection{Parallel Anytime SCAN}

Block processing. A key idea of anySCAN is processing vertices in blocks. Concretely, in Step 1, we choose $\alpha$ vertices for summarizing at each iteration $(\alpha \gg 1)$. Obviously, it increases the overlap between super-nodes. However, this overlap will lead to more super-nodes to be merged at Step 2 and will consequently reduce the number of structure similarity calculations in Step 3. Thus, the performance is improved. In Step 2 and 3, we choose $\beta$ vertices for updating at each iteration. Though this leads to more redundant similarity calculations, it reduces the overhead of the anytime scheme of anySCAN by reducing the number of iterations.

Block processing also allows threads to perform core checks, the most expensive part of anySCAN, concurrently, thus leading to a work-efficient parallel algorithm. Specifically, it maintains the anytime property in parallel mode.

Parallelizing. Figure 4 shows the pseudocode for anySCAN using OpenMP [18]. Since vertices have different neighborhood sizes, we use dynamic scheduling for load balancing, e.g., Line 6, 10, 30, and 34.

Step 1: Summarization. Please note that because two supernodes may overlap, assigning states for their shared vertices suffers may lead to a race condition, where threads could attempt to write different states for a vertice at the same time. To ensure consistency, a naive approach would need to lock the whole for loop (Line 9-14 Figure 2) for instance. However, this is clearly inefficient since this would sequentialize a large workload. To avoid that, we separate the for loop in Line 6 (Figure 2) into three different parts (Line 6-24 in Figure 4). First (Line 6-9), we calculate the neighborhood of all vertices $p$ and store the results in a temporary buffer $B$. We also mark the state of $p$ as described in Section 3.1. Obviously, vertices can be processed independently by threads. Second (Line 10-15), for each vertex $p$, we examine its neighbors $q$ and mark its state if $p$ is a core. If $q$ is a processed-noise or unprocessed-noise, it is marked as processedborder. If $\mathrm{q}$ is unknown, it is marked as unprocessed-border. Now, to check if $q$ is a core, we increase the number of neighbors of $q$ by 1 ( $q$ has $p$ as one of its neighbors) using an atomic operation (Line 14-15). If $q$ is an unprocessedborder and has more than $\mu$ neighbors, it will be marked as unprocessed-core. Last (Line 16-24), we use a sequential algorithm for storing the super-nodes as well as merging some of them as described in Section 3.1, since these tasks are highly sequential. However, the overall runtime of this part is negligible and has a small effect on the scalability of anySCAN as demonstrated in Section 5.2.

Step 2: Merging strongly-related super-nodes. As for Step 1 , we separate Step 2 into two parts for avoiding performance limiting synchronization. In Line 30-33, we perform the core check on each vertex $p$ in a selected subset of vertices $X$ independently using multiple threads and store the

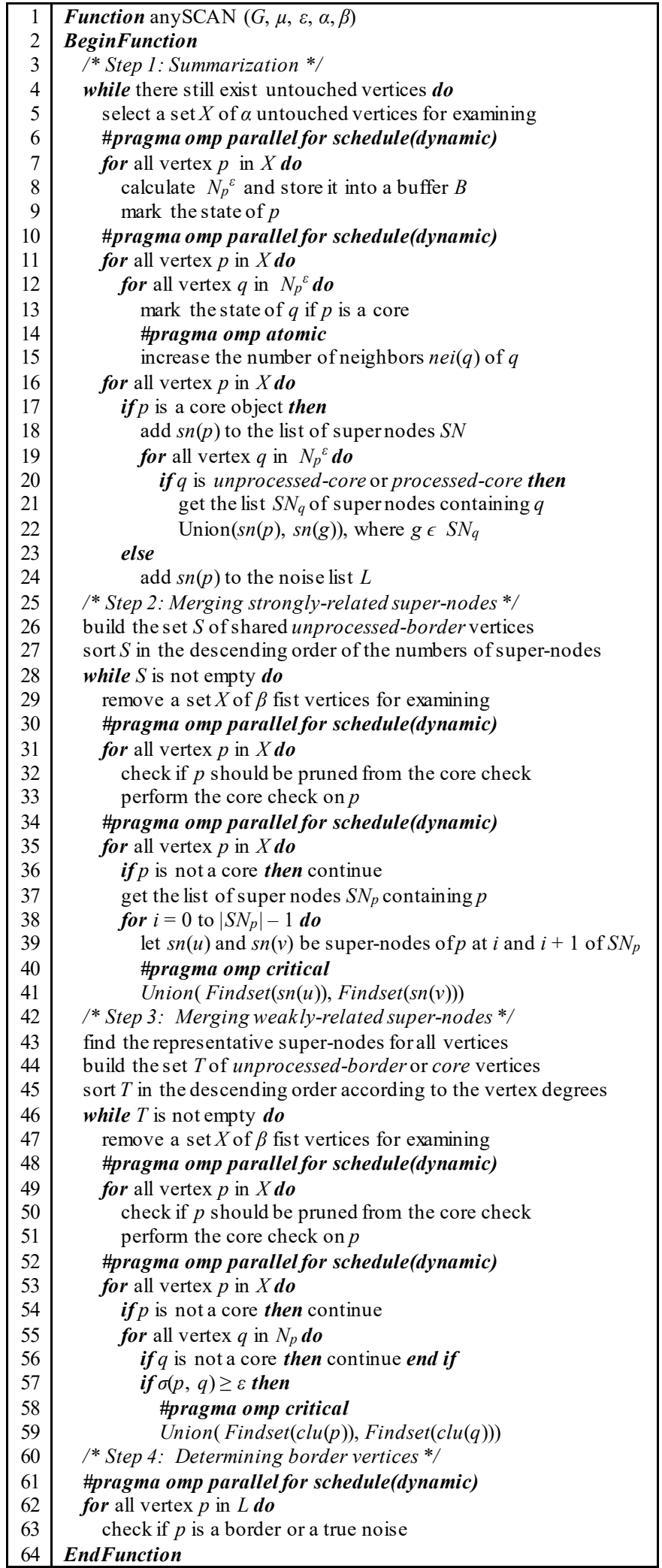

Fig. 4. Pseudocode for anySCAN using OMP

results. In Line 34-42, we check each vertex independently and merge related super-nodes when necessary as described in Section 3.1. The Union operation is not thread-safe. Thus, it must be locked inside critical sections (Line 41). However, since the number of super-nodes is much smaller than the number of vertices in our experiments, the number of Union operations is thus small and does not affect the scalability 
of anySCAN (see Figure 14 for more details). Note that, the pruning check needs to get the labels for vertices (which in turn is acquired from the labels of super-nodes via the Findset operation). Thus, if we did not split Step 2 into two parts, we would need to lock the Findset operation during the pruning check to ensure consistency. This would incur significant synchronization cost since the Findset operation is used more frequently than the Union operation.

Step 3: Merging weakly-related super-nodes. As for Step 2, we also separate Step 3 into two parts (Line 49 to 61). The first part is for checking the core properties of vertices, and the second part for merging super-nodes using Union.

Step 4: Determining border vertices. Each vertex $p$ inside the noise list $L$ can be examined independently to see if it is really an outlier (Line 63-65). Here some redundant calculation may happen if two noise vertices $p$ and $q$ share an unprocessed-border vertex due to the checking process in Step 4. However, this case very rarely happens in experiments. By accepting this, each vertex can be fully processed by a thread without having to wait for the results of other threads. Consequently, the scalability of anySCAN is improved.

\subsection{Algorithm Analysis}

Correctness. We first prove the correctness of anySCAN.

Lemma 5. The final results of anySCAN are identical to those of SCAN.

Proof 3. Assume that two vertices $p$ and $q$ belong to the same cluster in SCAN. There must exist a chain of core vertices $x_{1} \cdots x_{n}$ so that $p \triangleleft x_{1} \cdots \triangleleft x_{i} \triangleright \cdots \triangleright x_{n} \triangleright q$ (Definition 4 ). After Step 1, $x_{1}$ to $x_{n}$ must belong to some super-nodes $\operatorname{sn}\left(s_{1}\right) \cdots \operatorname{sn}\left(s_{m}\right)(m \leq n)$ since only unprocessed-noise or processed-noise vertices are excluded to the noise list $L$. In Step 2 and 3, if $\operatorname{sn}\left(s_{1}\right)$ to $s n\left(s_{m}\right)$ belong to different clusters, they will be connected by some other vertices or by some $x_{i}(1 \leq i \leq n)$ themselves (Lemmas 3 and 4). Thus, there exists a density-connected path from $x_{1}$ to $x_{n}$ through $\operatorname{sn}\left(s_{1}\right)$ to $\operatorname{sn}\left(s_{m}\right)$ (some $x_{i}$ may not be included in the path because they are not processed). Similarly, Step 4 guarantees that if $p$ and $q$ are in $L$, they are still density-connected to $x_{1}$ and $x_{n}$ by some paths. Thus, $p$ and $q$ are also density-connected in anySCAN. Note that, in both anySCAN and SCAN, a shared-border vertex may be assigned to different clusters according to the examining order of vertices.

Complexity analysis. Let $S N$ be the set of super-nodes, $T_{p q}=\min \left(\left|N_{p}\right|,\left|N_{q}\right|\right)$ be the time for computing $\sigma(p, q)$, and $c$ a constant. The time complexity of Step 1 is $O\left(\sum_{s n(p) \in S N \cup L}\left(\sum_{q \in N_{p}} T_{p q}+\sum_{q \in N_{p}} c\right)\right)$ for calculating neighborhoods and marking the states of vertices. We have $O\left(\sum_{q \in C}\left|N_{q}^{\epsilon}\right|\right)$ Union-Find operations (since $S N(q) \subset$ $N_{q}^{\epsilon} \subset N_{q}$ and each merge is done on new super-nodes only), where $C \subset V$ is the set of core vertices. Similarly, Step 2 and 3 need $O\left(\sum_{p \in S}\left(\sum_{q \in N_{p}}+\sum_{q \in N_{p}} T_{p q}\right)\right)$ time for core checks and neighborhood calculations and $O\left(\sum_{p \in S}\left|N_{p}\right|\right)$ Union-Find operation. Step 4 needs $O\left(\sum_{p \in L} \sum_{q \in N_{p}} T_{p q}\right)$ time for checking border states. Obviously, all of Steps 1 to 4 are bounded by $O\left(|E| T_{p q}\right)$ neighborhood calculations and $O(|E|)$ Union-Find operations. Sorting requires $O(|V|)$ time using bin-sort since the values are all integer and are bounded by the vertex degrees. Thus, at the end, anySCAN has the same time complexity as pSCAN and SCAN with $O\left(\min \left(N_{p}, N_{q}\right)|E|+|E| f(|V|)\right)$, where $f()$ is the extremely slowly growing inverse of the single-valued Ackermann function [5], [17]. The algorithm anySCAN needs to store the super-node list $S N$ as well as the noise list $L$. Thus, it incurs additional memory usage compared to SCAN. However, the overall size is still bounded by $O(|E|)$. Thus, in the end, it has $O(|V|+|E|)$ space complexity.

\subsection{Optimizations}

We introduce some optimization techniques to speed up the similarity calculation of anySCAN inspired by [5].

Lemma 6. Given two vertices $p$ and $q$, if $\hat{\sigma}(p, q)^{2}<\epsilon^{2}$. $l_{p} \cdot l_{q}$, where $\hat{\sigma}(p, q)=\min \left(\left|N_{p}\right|,\left|N_{q}\right|\right) \cdot \max \left(w_{p}, w_{q}\right)$, and $w_{p}=\max _{q \in N_{p}}\left(w_{p q}\right)$, and $l_{p}=\sum_{r \in N_{p}} w_{p r}^{2}$, then $\sigma(p, q)<\epsilon$.

Proof 4. We have $\sum_{r \in N_{p} \cap N_{q}} w_{p r} \cdot w_{q r} \leq \min \left(\left|N_{p}\right|,\left|N_{q}\right|\right) \cdot$ $\max \left(w_{p}, w_{q}\right)<\epsilon \cdot \sqrt{l_{p} \cdot l_{q}}$. Thus $\sigma(p, q)=$ $\left(\sum_{r \in N_{p} \cap N_{q}} w_{p r} \cdot w_{q r}\right) / \sqrt{l_{p} \cdot l_{q}}<\epsilon$

The equation $\hat{\sigma}(p, q)^{2}<\epsilon^{2} \cdot l_{p} \cdot l_{q}$ can be verified in $O(1)$ time (since $w_{p}$ and $l_{p}$ are fixed and can be calculated in a preprocessing step in $O\left(\left|N_{p}\right|\right)$ time). If it is true, we do not need to calculate the structural similarity $\sigma(p, q)$. Moreover, while calculating $\sum_{r \in N_{p} \cap N_{q}} w_{p r} \cdot w_{q r}$, if the intermediate result is bigger than $\sqrt{\epsilon^{2} \cdot l_{p} \cdot l_{q}}$, then $\sigma(p, q)$ is bigger than $\epsilon$. Thus, we can stop for further reducing runtime.

\section{Dynamic Graph Clustering}

Given a weighted dynamic graph $G$, possible changes in $G$ are deletion, insertion and weight change on each edge. A naive approach would perform clustering from scratch, which is obviously very expensive. Here, we focus on an efficient incremental approach. Let $U=\{(u, v, w, t)\}$ be a set of changed edges $(u, v, w)$, where $t$ is the update type including insertion, deletion and change weight. Instead of updating clusters with each change [8], [19], [20], our method, called dynamic anySCAN (danySCAN), operates in a bulk scheme, especially when the number of updates $|U|$ is large. For simplicity, we skip $w$ and $t$ whenever it is clear from the context.

\subsection{Dynamic Anytime SCAN}

The general idea of danySCAN is that we exploit the existing super-node structure for incrementally updating clusters without having to start from scratch. We first present the general process of danySCAN with arbitrary graph updates before going into the optimization procedure of each special kind of update operations.

Definition 8. Given a set of updates $U$, the set of directly affected vertices $A$ is defined as a set of vertices in $U$.

Definition 9. Given a set of updates $U$, the set of affected edges $B$ is defined as a set of all edges $(u, v)$ where $u \in A$ and/or $v \in A$.

Definition 10. The set of vertices $A^{k}$ is called the $k$-affected of $A$ if it consists of all vertices that are $k$-hop-away from any vertices in $A$. 


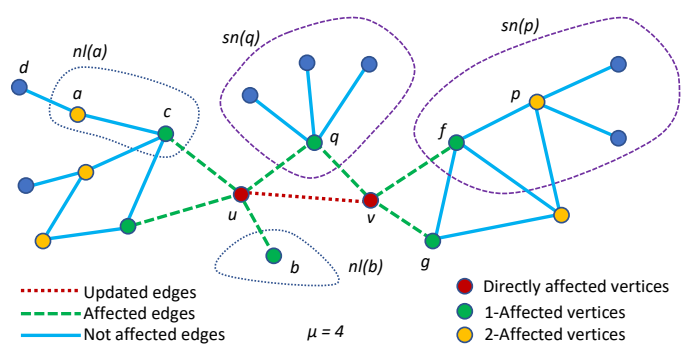

Fig. 5. Affected vertices, edges, super-nodes and noises w.r.t. a changed edge $(u, v) \in U$

Lemma 7. All edges $(u, v)$ in $B$ will change their structural similarities after the update.

Lemma 7 is directly inferred from Definition 1. Following it, all 1-affected vertices in $A^{1}$ may change their core properties since their structural neighborhoods will be changed. Conversely, all edges $e \in E \backslash B$ will not be affected. Their similarities remain the same after the updates. Similarly, all vertices $v \in V \backslash A^{1}$ will not change their core properties.

Figure 5 shows an example when we change (e.g., delete) an edge $(u, v)$. The structural similarity $\sigma(u, c)$ will be changed. Thus, vertex $c$ may change its core properties. However, vertices $a$ and $d$ are not affected at all. Their core properties remain the same. Each super-node $s n(q)$ where $q \in A^{1}$ will also be affected by the updates following the similarity changes of $e \in B$. Some vertices may be removed from $\operatorname{sn}(q)$ or inserted into $\operatorname{sn}(q)$, e.g., $u$ or $v$. If $|\operatorname{sn}(q)|<\mu$, the super-node must be removed since $q$ is not a core vertex anymore. We call $s n(q)$ a directly affected super-node. For $\operatorname{sn}(p)$ where $p \in A^{2} \backslash A^{1}$, since $N_{p}^{\epsilon}$ is not affected, its members will not change. However, since vertex $f \in \operatorname{sn}(p)$ might change its core property, it will affect the connectivity between $\operatorname{sn}(p)$ and its nearby super-nodes, thus leading to changes of current clusters. We call $s n(p)$ an affected supernode. All other super-nodes outside the 2-affected set will not affect the cluster structure at all since all of their vertices do not change after the updates. Similarly, a noise vertex $n l(b) \in L$ is called a directly affected noise if $b \in A^{1}$ since its members can be changed into core vertex or border vertex of another cluster. A noise object $n l(a)$ is an affected-noise if $a \in A^{2} \backslash A^{1}$. Even though its members remain the same, it may be assigned to another cluster following the change of the core property of one of its members, e.g., vertex $c$.

The algorithm danySCAN consists of several steps as shown in Figure 6 and described below. Generally, it will update the super-node structure w.r.t. updates before merging them again to build clusters.

Step 1. Updating the super-node structures. When changes occur, we need to rebuild the list of super-nodes and the noise list $L$ as well as their relationships.

First, we need to update the graph $G$ to reflect the changes and then recalculate the lengths of vertices for speeding up similarity evaluations (Line 4-5 in Figure 6).

Second, we need to check the state of each affected edge $e \in B$ (Line 6-8). To do so, we assign for each edge $(u, v) \in E$ a state including: yes if $\sigma(u, v) \geq \epsilon$, no if $\sigma(u, v)<\epsilon$ and unknown if $\sigma(u, v)$ has not been calculated yet. Now, for simplicity, we can set all edges $e \in B$ to the unknown state, indicating that their similarities are changed

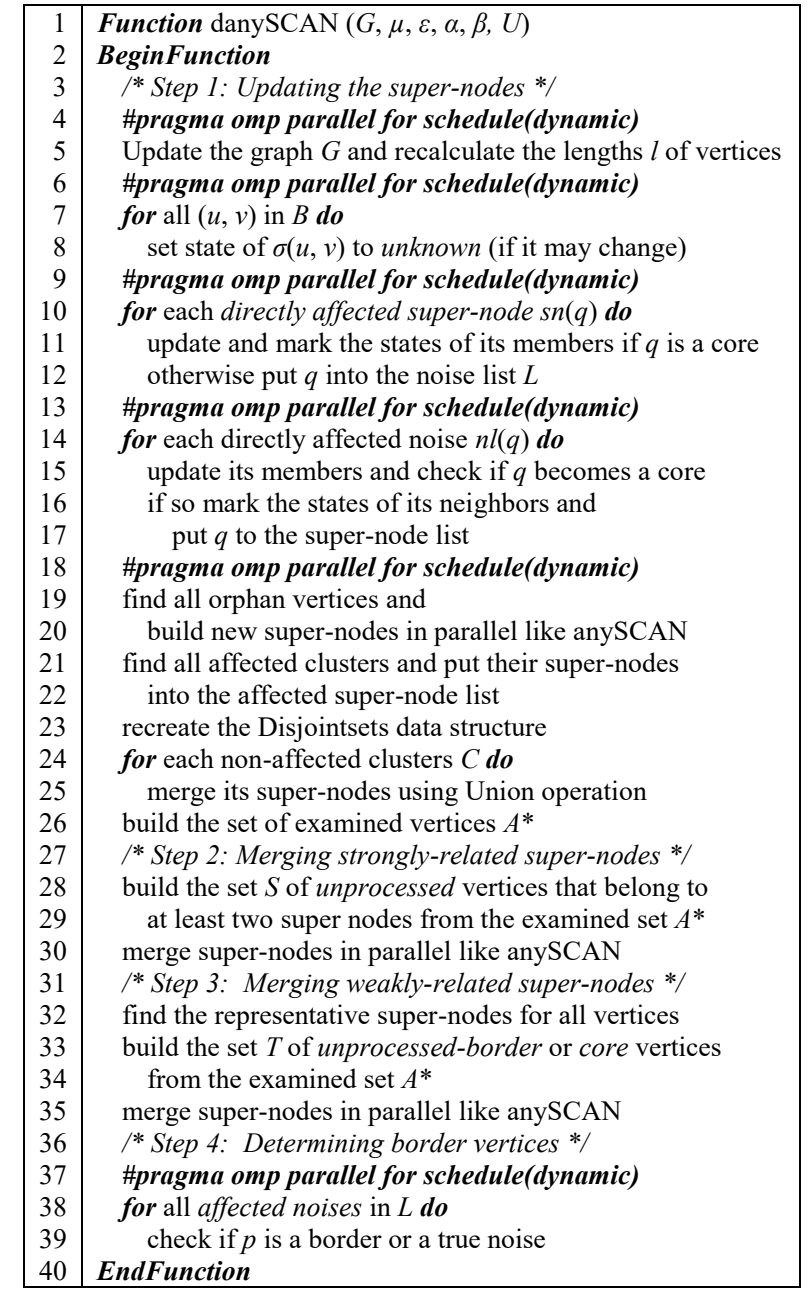

Fig. 6. Pseudocode for dynamic anySCAN

and need to be recalculated. However, for efficiency, we can use some optimization techniques for different kinds of update operations as presented in Section 4.2.

Third, we set the states of all vertices $p \in A$ and $p \in$ $\operatorname{sn}(q)$, where $s n(q)$ is a directly affected super-node to unknown. Then, for each directly affected super-node $\operatorname{sn}(q)$, we update its members by adding a new vertex $r$ if $\sigma(q, r) \geq \epsilon$ or removing $r$ if $\sigma(q, r)<\epsilon$. If $\mid s n(q)<\mu$, we delete $\operatorname{sn}(q)$ from the list of super-nodes and move it to the noise list $L$. Otherwise, we mark the state of $p \in \operatorname{sn}(q)$ following the transition state diagram in Figure 3 like for Step 1 of anySCAN (Line 9-12). Similarly, for each directly affected noise vertex $n l(q) \in L$, we update its members. If $q$ is a core, we put $\operatorname{sn}(q)$ into the super-node list and mark the states of its neighbors accordingly (Line 13-17). At the end of this stage, if a vertex $q$ is still an orphan (i.e., state $(q)=$ unknown), we check if it actually belongs to some unaffected super-nodes. If so, $q$ is marked as unprocessed-border.

Fourth, we build new super-nodes and new noise vertices over orphan vertices in a similar way to the summarization step of anySCAN described in Section 3.1, which we will not repeat here for clarity, until there are no more unknown ones (Line 19-20). After that we further extend the sets of affected super-node and affected noises by adding newly generated ones to them.

Fifth, in the worst case, an affected super-node $s n(p)$ may lose its connections to other super-nodes due to the 
changes of core properties of its members or one of its yes connections to others is broken. This may split its cluster into smaller groups. Thus, this cluster needs to be re-verified to identify sub-clusters. Note that, we also have merge cases where clusters are merged into a larger one. But, these cases are easier due to the merging nature of anySCAN as presented in Section 3.1. Here a cluster $C$ is an affected cluster if one of its super-nodes is affected. For each affected cluster, we put its super-nodes into the affected super-node list for rebuilding sub-clusters later (Line 21-22).

Seventh, we need to rebuilt the cluster structure if there is a split case. To do so, we recreate a new Disjointsets (Line 23). Then, for each non-affected cluster $C$ with $c$ super-nodes, we merge them by calling the Union operation exactly $c-1$ times for each adjacent pair for rebuilding $C$ in the new Disjointsets (Line 24-25). Note that since the number of super-nodes is much smaller than the number of vertices, this task thus has negligible effect on the performance of danySCAN. Now we need to identify a special set of vertices $A^{*}$ called examined vertices as follows before fully building final clusters in the next steps of danySCAN (Line 26).

Definition 11. The set of examined vertices $A^{*}$ consists of all vertices in $\cup s n(p)$, where $s n(p)$ is an affected super-node and all vertices in $A^{2}$.

Since we need to merge affected super-nodes to form clusters in the next steps, it is straightforward to see that we only need to examine vertices in $A^{*}$ for efficiency. $A^{2}$ ensures that super-nodes are merged due to the update. And $\cup \operatorname{sn}(p)$ guarantees that if a cluster is not broken, it will be recreated correctly. When the number of updates is large, $A^{*} \approx V$. Thus, we can choose $A^{*}=V$ for simplicity.

Step 2: Merging strongly-related super-nodes. This step (Line 27-30) works in a similar way to anySCAN with some major modifications: (1) we only consider objects in the examined set $A^{*}$ and (2) we consider both unprocessedborder and unprocessed-core since we have not processed unprocessed-core in Step 1 (Line 27-30).

Step 3: Merging weakly-related super-nodes. This step (Line 31-35) also works exactly as in anySCAN with a restriction on the examined set $A^{*}$ (Line 31-35).

Step 4: Determining border vertices. It is easy to prove that if a noise vertex $p \in L$ is not an affected noise, it will never be changed. Note that if $p$ is a shared border vertex, it may be assigned different labels according to the examining order. Thus, we only take out all affected noises and examine them using the same procedure of anySCAN.

\subsection{Optimization techniques for danySCAN}

The above procedure of danySCAN works regardless of the update types. However, for efficiency, we can reformulate the second phase of Step 1 to cope with different update types by exploiting some similarity check as in [8]. For example, if an edge $(u, v)$ is inserted, for each vertex $w \in N_{u} \backslash N_{v}, \sigma(w, u)$ will be decreased. Thus, if state $(u, v)=n o$, it will never change to yes. Thus, we do not need to re-evaluate the structural similarity between $u$ and $v$ anymore. We can update edge states in Step 1 in two ways: (1) sequentially with each updates which incurs redundant calculations when several updated edges share a vertex; or

\begin{tabular}{|c|r|r|r|r|}
\hline Graph & Vertices & Edges & $\bar{d}$ & $\mathrm{c}$ \\
\hline Ego-Gplus (GR01) & 107,614 & $13,673,453$ & 127.06 & 0.4901 \\
Soc-LiveJournal1 (GR02) & $4,847,571$ & $68,993,773$ & 14.23 & 0.2742 \\
Soc-Poket (GR03) & $1,632,803$ & $30,622,564$ & 18.75 & 0.1094 \\
Com-Orkut (GR04) & $3,072,441$ & $117,185,083$ & 38.14 & 0.1666 \\
Kron_g500-logn21 (GR05) & $2,097,152$ & $182,082,942$ & 86.82 & 0.1649 \\
Twitter (GR06) & $41,652,230$ & $1,369,000,750$ & 32.8 & 0.0730 \\
\hline
\end{tabular}

TABLE 1

REAL GRAPH DATASETS ( $\bar{d}$ IS AVERAGED VERTEX DEGREES AND $c$ IS AVERAGED CLUSTER COEFFICIENTS)

(2) check all affected vertices in chunks for avoiding shared vertices. In the latter case, we must modify the similarity check of [8] for ensuring the correctness of the algorithm by only considering $w \in N_{u} \backslash N_{v}$ if $w$ is not an affected vertex as in Lemma 8 below. However, compared to (1), (2) may have weaker pruning power in case we have big chunks.

Lemma 8. Given an inserted edge $(u, v)$, for each vertex $w \in$ $N_{u} \backslash N_{v} \wedge N_{w} \cap A=\emptyset, \sigma(u, w)$ will be decreased.

Proof 5. Since $N_{w} \cap A=\emptyset$, its length $l_{w}$ is not changed while $l_{u}$ is increased due to the insertion. Thus, $\sigma(u, w)$ is decreased following Definition 1.

Here, we introduce two more cases to cope with weighted graphs: (1) increase weight which is similar to the edge insertion and (2) decrease weight which is similar to the edge deletion in [8]. The detailed extension for all update types is straightforward but lengthly, and thus is omitted.

\subsection{Dynamic AnySCAN on Multicore CPUs}

The extension of danySCAN for multicore CPUs is exactly the same as anySCAN with the block processing scheme in the fourth phase of Step 1, Step 2 and 3. The pseudocode of danySCAN can be found in Figure 6.

\subsection{Algorithm Analysis}

Correctness. We first prove the correctness of danySCAN.

Lemma 9. The final results of danySCAN are identical to those of SCAN.

Proof 6. In Step 1, danySCAN checks each super-node to add or remove members. Each new super-node $p$ is also built by a neighborhood query. Thus, this guarantees that $\forall s n(p): s n(p)=N_{p}^{\epsilon}$. Step 1 also ensures that none of the vertices in $L$ is actually a core. Consequently, if two vertices $p$ and $q$ are density-connected in SCAN by a chain of core vertices $x_{1} \cdots x_{n}$ (Definition 4 ), all $x_{i}$ must belong to some super-nodes $\operatorname{sn}\left(s_{j}\right)$ after Step 1 of danySCAN. At the end of Step 1, all possibly changed connections among super-nodes are removed to check them again in Step 2 and 3. Following Lemmas 3 and 4, all $s n\left(s_{j}\right)$ will be put into the same cluster via $x_{i}$ or other core vertices. Due to Lemma 2, $p$ and $q$ are connected via a chain going through $\operatorname{sn}\left(s_{j}\right)$ (including $s_{j}$ itself). Step 4 guarantees that if a border vertex is added to the noise list $L$ initially, it is surely discovered as a border here. Thus, the results of danySCAN and SCAN are identical.

Complexity analysis. The time complexity of danySCAN is similar to that of anySCAN, pSCAN and SCAN. It also consumes $O(|E|+|V|)$ space like others. 

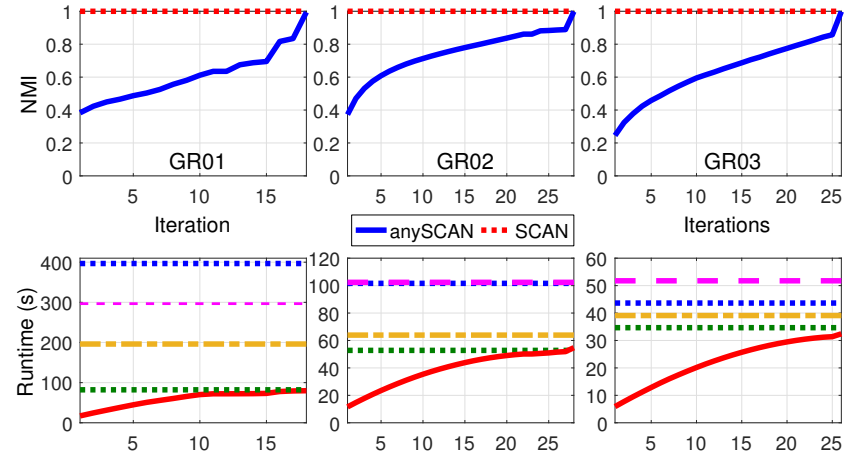

$\begin{array}{rrr}\text { Iteration } & \text { Iterations } \\ \text { - anySCAN } \ldots \mathrm{SCAN}_{++} & \cdots \mathrm{pSCAN}\end{array}$

Iterations

- SCAN - -SCAN-B

Fig. 7. NMI scores and cumulative runtimes of anySCAN during its execution in comparison with other batch algorithms (represented by horizontal lines) for GR01 to GR03

\section{EXPERIMENTS}

All experiments are conducted on a Linux workstation with two 3.1 GHz Intel Xeon CPUs with 64 GB local RAM each using g++ 4.8.3 (-O3 flag) and OpenMP ${ }^{2}$ 3.1. We use 6 large datasets (see Table 1) acquired from the Stanford Network Analysis Project (SNAP) ${ }^{3}$ [21], The UF Sparse Matrix Collection $^{4}$, and the Laboratory of Web Algorithmics ${ }^{5}$ [22].

\subsection{Anytime SCAN}

We compare anySCAN with the original algorithm SCAN and its fastest variants pSCAN [5] and SCAN++ [4]. Since these state-of-the-art techniques are originally designed to work with unweighted graphs, we extend them to work with weighted ones as described in Section 2. We also study SCAN-B, our extension of SCAN using the optimization techniques described in Section 3.4. For evaluating the anytime property of anySCAN, we use the results of SCAN as ground truth and Normalized Mutual Information (NMI) scores [23] for assessing how close the intermediate result is compared to that of SCAN. NMI is defined as the geometric mean of shared information between the clustering result $C$ and the ground truth $T$ and their conditional entropy. Its score is in $[0,1]$ where 1 means both results are identical. Unless otherwise stated, we use default parameters $\mu=5$, $\epsilon=0.5$, and $\alpha=\beta=8192$. Due to space limitations, we omit some graphs here. Interested readers please refer to [7] for more results on all graphs.

Anytime properties. Figure 7 shows the cumulative runtimes and NMI scores of anySCAN for GR01 to GR03 (see Table 1) measured at different iterations of Steps 1 to 3 of anySCAN. As we can see, the clustering qualities of anySCAN improve over time and converge toward the results of SCAN at the end (indicating by $N M I \approx 1.0$ ). The longer it is run, the better the NMI scores it obtains, i.e., its results are more similar to those of SCAN. Additionally, since anySCAN is an anytime algorithm, it has the benefit that it can be stopped at arbitrary time points for approximating results as well as saving computation cost. For example, one can stop anySCAN with good NMI

2. http://www.openmp.org/

3. https://snap.stanford.edu/

4. http:/ / www.cise.ufl.edu/research/sparse/matrices/

5. http://law.di.unimi.it/datasets.php

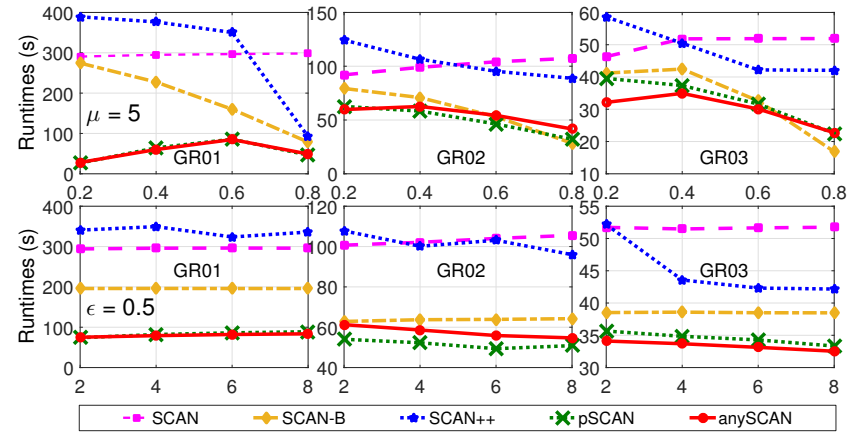

Fig. 8. Final runtimes of different algorithms w.r.t. parameters $\epsilon$ (top) and $\mu$ (bottom) for GR01 to GR03

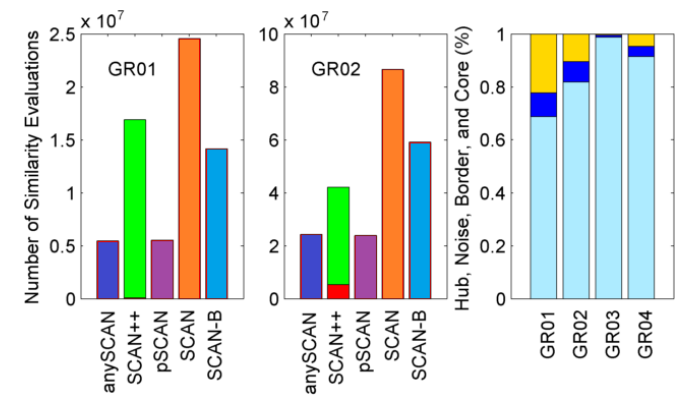

Fig. 9. (Left) Numbers of structural similarity calculations for all algorithms. For SCAN++, numbers of true similarity (bottom) and similarity sharing (top) evaluations are plotted. (Right) Numbers of hub and outlier (light blue), border (dark blue), and core (yellow) vertices (from bottom to the top) for some datasets

scores $\approx 0.5$ after $24.08,13.94$, and 8.91 seconds for GR01 to GR03 and acquire acceleration factors of up to 14.55 times compared to SCAN. This property makes anySCAN an interactive algorithm that fits well in systems with limited time constraints and that require fast response times.

Overall performance. In Figure 8, we further compare the final cumulative runtimes of anySCAN and others w.r.t. parameters $\mu(\epsilon=0.5)$ and $\epsilon(\mu=5)$ for GR01 to GR03 (GR04 to GR05 are omitted due to space constraints). pSCAN is slightly faster than anySCAN on GR02 $(\bar{d}=14.2)$ and GR05 $(\bar{d}=15.8)$, and is slightly slower than anySCAN on GR01 $(\bar{d}=127.0)$, GR03 $(\bar{d}=18.7)$, and GR04 $(\bar{d}=38.1)$. SCAN++ does not work well when $\epsilon$ and $\mu$ are small due to its two-hop-away-node (DTAR) expansion scheme. The bigger the number and the neighborhood sizes of core vertices, the more structural similarity evaluations it must perform, thus making SCAN++ slower than SCAN in some cases due to its additional overhead of calculating and maintaining the DTARs. Similar results are also observed in [5]. Interestingly, SCAN-B works quite well despite its simplicity. For sparse graphs, e.g. GR02 and GR03, and high values of $\epsilon$, e.g. $\epsilon=0.8$, it is sometimes slightly faster than pSCAN and anySCAN. The reason is that most structure similarity calculations are skipped due to the filtering property described in Lemma 6, especially when $\epsilon$ is very high. For GR06 with 1.3 billion edges $(\mu=5, \epsilon=0.5)$, pSCAN and anySCAN need 2806.49 and 2770.94 seconds, respectively, while SCAN cannot finish after 12 hours.

Similarity evaluations. Figure 9 shows the number of structural similarity evaluations for all algorithms and thus further clarifies the acquired results above. For all datasets, 

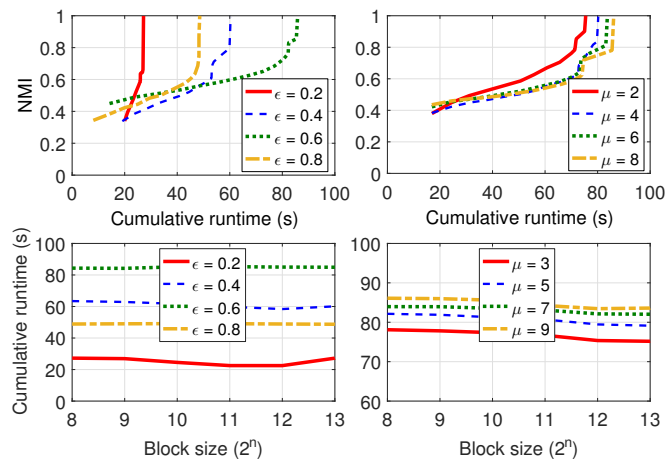

Fig. 10. The effect of parameters $\mu$ and $\epsilon$ (left) and block sizes $\alpha=\beta$ (right) for GR01

pSCAN and anySCAN use almost the same number of similarity calculations, which is much smaller than those of other methods. The number of similarity sharing calculations of SCAN++ is clearly correlated with the numbers of core vertices. The higher the number of core vertices, the more similarity sharing SCAN++ uses, meaning that the similarity evaluation time will be reduced. However, it also means that SCAN++ incurs more overhead for expanding its DTAR clusters. Sometimes, this overhead surpasses the similarity sharing benefit, thus making SCAN++ slower than SCAN-B (even though it uses fewer calculations), e.g. on GR02.

Parameter analysis. The effect of parameter $\epsilon$ on anySCAN is shown in Figure 10 (top). Due to its summarization scheme, too small $\epsilon$, e.g. 0.2 , means many super-nodes are created earlier, thus leading to better approximate results earlier. Too high $\epsilon$, e.g. 0.8 , creates many noise vertices in the beginning, thus making NMI higher (than medium values of $\epsilon$ ) since they could be regarded as members of a special cluster. In contrast to $\epsilon$, the effect of $\mu$ is quite straightforward: lower values of $\mu$ mean better approximate results since there are more core vertices to be discovered at each iteration of anySCAN. This means super nodes are merged earlier and thus makes anySCAN reach the final result of SCAN faster.

The effect of block size parameters $\alpha$ and $\beta$ is also clear in Figure 10 (bottom). Too small values make anySCAN slower due to its anytime overhead at each iteration. When we increase the block size, there are more super-nodes. Their overlap helps to reduce the runtime by connecting more super-nodes earlier, thus reducing the number of similarity evaluations at Step 2 and Step 3 of anySCAN. For example, with $\mu=4$, anySCAN decreases from 82.1 to 80.6 and 79.1 seconds when $\alpha=\beta$ increase from 256 to 2048 and 8192, respectively. However, when $\alpha=\beta$ are too large, redundant similarity calculations may appear during Step 1 . Thus, the runtime of anySCAN may slightly increase. For example, with $\epsilon=0.2$, anySCAN requires 27.2, 22.5 seconds, and 28.1 seconds when $\alpha=\beta=256,2048$, and 8192, respectively. The changes, however, are very small. This means that the performance of anySCAN is stable w.r.t. the block sizes.

Performance on synthetic graphs. Table 2 summarizes some synthetic graphs created by LFR bench mark graphs [24]. We set the number of vertices to $1,000,000$ and vary the number of edges in terms of average vertex degrees and average cluster coefficients. The maximum degree is set to 100 .

Figure 11 shows the performance of pSCAN and

\begin{tabular}{|c|c|r|r|r|}
\hline Id & Vertices & Edges & $\bar{d}$ & $\mathrm{c}$ \\
\hline LFR01 & $1,000,000$ & $22,283,773$ & 44.567 & 0.4017 \\
LFR02 & $1,000,000$ & $25,064,820$ & 50.129 & 0.4007 \\
LFR03 & $1,000,000$ & $27,599,929$ & 55.199 & 0.4022 \\
LFR04 & $1,000,000$ & $29,937,286$ & 59.874 & 0.4011 \\
LFR05 & $1,000,000$ & $32,527,885$ & 65.055 & 0.4004 \\
\hline LFR11 & $1,000,000$ & $25,064,820$ & 50.129 & 0.2012 \\
LFR12 & $1,000,000$ & $25,064,820$ & 50.129 & 0.3029 \\
LFR13 & $1,000,000$ & $25,064,820$ & 50.129 & 0.4168 \\
LFR14 & $1,000,000$ & $25,064,820$ & 50.129 & 0.5012 \\
LFR15 & $1,000,000$ & $25,064,820$ & 50.129 & 0.6003 \\
\hline
\end{tabular}

TABLE 2

SYNTHETIC GRAPH DATASETS $(\bar{d}$ IS AVERAGED VERTEX DEGREE AND $c$ IS AVERAGED CLUSTER COEFFICIENT)
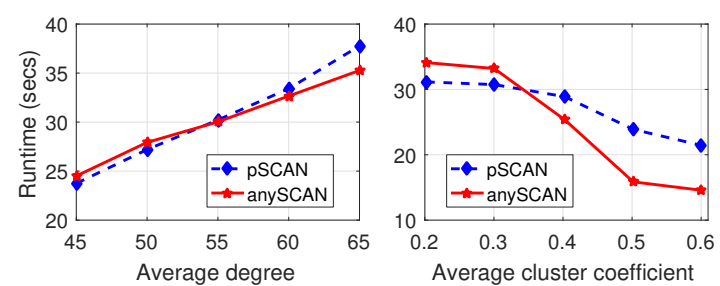

Fig. 11. Performance on synthetic graphs
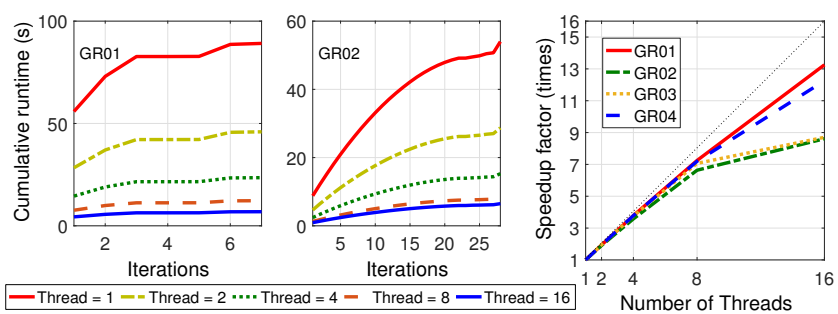

Fig. 12. Cumulative runtimes of anySCAN after each iteration of its anytime scheme for different numbers of threads (left) and the final runtime scalability (right)

anySCAN on these synthetic graphs. When the number of edges (indicated by the average vertex degree) increases, the runtimes of both algorithm increase since more structural similarity needs to be computed. However, anySCAN tends to perform better than pSCAN on denser graphs. When the average cluster coefficient increases from 0.2 to 0.6 the runtimes of both methods decreases. Again, anySCAN tends to perform better than pSCAN on datasets with higher average cluster coefficients. This can be explained by the way anySCAN performs clustering. The denser the graphs and the better separated cluster structures, the more vertices will be put in each super-node, thus reducing the efforts for connecting them together. This leads to an improvement of the overall performance.

\subsection{Multicore Anytime SCAN}

Anytime properties. Since anySCAN is an anytime and parallel algorithm at the same time, we study how it scales with the number of threads. As shown in Figure 12 (left), anySCAN scales very well for each iteration of its anytime scheme, using $\alpha=\beta=32768$ as a default parameter in this section. For GR01, the speedup factors at all examined time points are very high (around 13.25 for 16 threads). More interestingly, for most datasets, the scalability of anySCAN slightly declines at each iteration. For GR02 and 16 threads as an example, at the first iteration, it achieves 9.52 times speedup factor. However, at the end, the speed up factor 

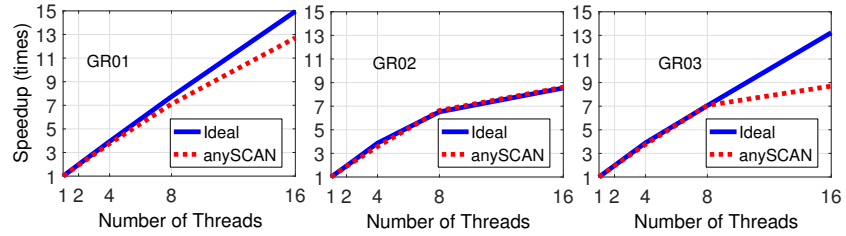

Fig. 13. Speed up factors of anySCAN and an ideal algorithm w.r.t. different numbers of threads

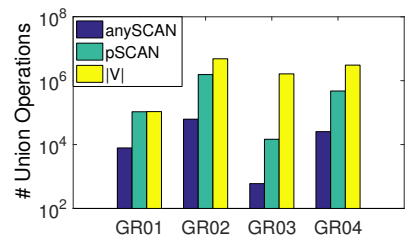

Fig. 14. Number of performed Union operations for GR01 to GR04

reduces to 8.61. Thus, the earlier a user stops the algorithm, the higher the speed up factor she enjoys.

Figure 12 (right) shows the final speedup factors of anySCAN w.r.t. different numbers of threads for GR01 to GR04 (GR05 and GR06 are omitted for clarity). anySCAN scales very well with the numbers of threads, e.g., almost linearly for GR01, GR04, and GR05. For GR01, the speedup factors over single thread are 1.93, 3.78, 7.24, and 13.25 for $2,4,8$, and 16 threads, respectively. Using 16 threads, the speed up factors are 12.25, 11.41 and 9.35 for GR04, GR05 and GR06, respectively. The speed up factors on GR02 and GR03 are worse than those of the others. Beside the common NUMA effect (threads are run on two different CPUs with 64 GB local memory each), one reason clearly is the sparseness of the graph. GR02 and GR03 are much sparser than GR01, GR04 and GR05 (indicated by the averaged vertex degrees $\bar{d}$ ). Moreover, the degrees of vertices vary significantly on GR02 and GR03. These make the workloads of threads very unbalanced, thus reducing the scalability of anySCAN (see also Figure 13 for further analysis). In this case, increasing the block size values will help to solve the problem (see Figure 15 for the parameter analysis). Sorting vertices and processing ones with higher degrees first might also balance the workloads better.

Performance comparison. Since anySCAN is the first parallel version of SCAN on multicore processors, we compare it with an ideal parallel algorithm for further assessing its performance in Figure 13. The ideal algorithm only calculates the structural similarities (without optimizations) of all edges of $G$ which is the most expensive part of SCAN and ignores the label propagation process among vertices to build clusters. Obviously, it does not require synchronizations among threads and thus has an ideal scalability w.r.t. the number of threads. Its performance is calculated by the speedup factor between the single thread and multiplethread usages. Note that pSCAN [5] and SCAN++ [4] are highly sequential and are non-trivial problems for parallelizing efficiently. As we can see, anySCAN acquires very close performance to the ideal algorithm for most datasets, especially GR02. For GR03, the performance difference between anySCAN and the ideal method is larger than for the other data sets for 16 threads. Here, optimization techniques for speeding up the structural similarity calculation cause the problem. Most calculations are filtered out earlier following
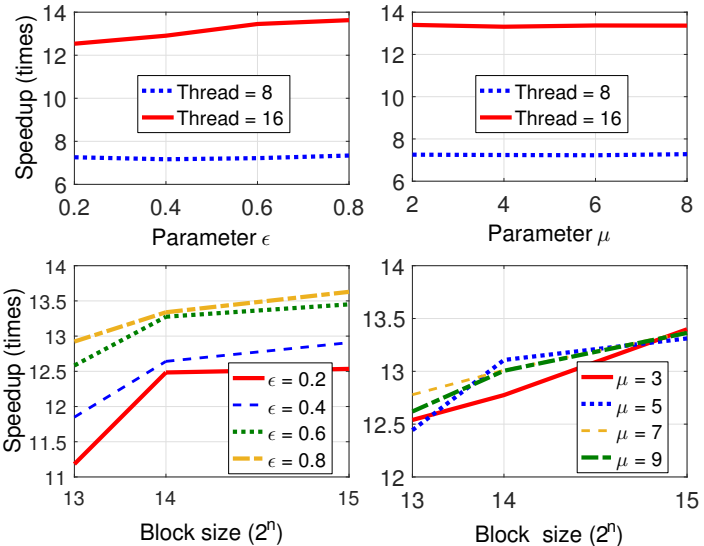

Fig. 15. The effect of parameters $\mu, \epsilon$, and block sizes on the scalability of anySCAN for the dataset GR01
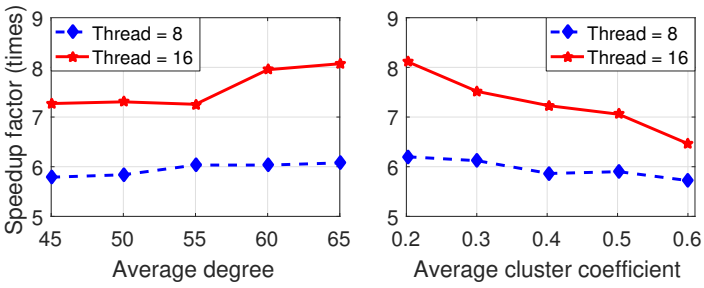

Fig. 16. Scalability on synthetic graphs

Lemma 6. Even though this makes the algorithm much more efficient, it lowers the overall workload for threads at each iteration of anySCAN, thus making it more sensitive to the load balancing problem, as well as reducing the ratio of sequential and parallel parts of anySCAN and thus the scalability following Amdahl's law for scalability.

AnySCAN needs to perform the Union operations inside critical areas for merging super-nodes inside Step 2 and 3. Thus, the numbers of Union operations strongly affect its scalability and are shown in Figure 14. Since pSCAN uses the Disjoint Set data structure like anySCAN, we include it here for a comparison even though it is not a parallel algorithm. As we see, pSCAN uses much fewer Union operations than the numbers of vertices $|V|$ of $G$. And, anySCAN uses even fewer operations (up to 25 times and 2725 times compared to pSCAN and $|V|$, respectively). Moreover, most of them $(7685 / 7844,31440 / 62351,268 / 599$, and 19969/25426 operations for GR01 to GR04, respectively) are executed sequentially in Step 1 of anySCAN, leaving only few to be executed in Step 2 and 3 inside critical sections. And fewer operations means better scalability of anySCAN as shown in Figure 12.

Parameter analysis. Processing times of different core vertices vary significantly depending on their neighborhood sizes, and are obviously more expensive than those of noise ones. Balancing the workloads for threads is therefore harder if there are more core vertices. Moreover, the number of merged super-nodes is higher. Thus, increasing $\mu$ and $\epsilon$ will lead to greater speed up factors as shown in Figure 15 (top), since the number of core vertices is reduced. On the other hand, increasing the block size will provide more work for threads at each iteration, therefore increasing the workload balance and thus increasing the scalability of anySCAN as shown in Figure 15 (bottom).

Performance on synthetic graphs. Generally, when the 


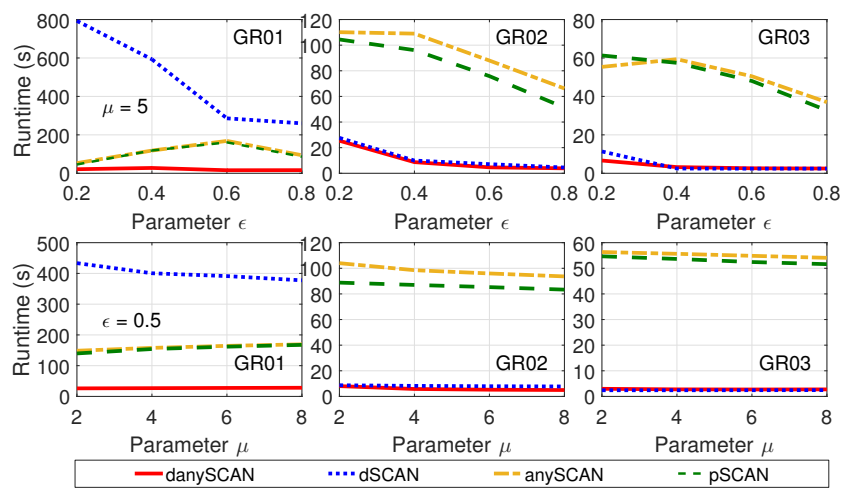

Fig. 17. Performance of danySCAN and others for GR01 to GR03 with different parameters $\epsilon(\mu=5)$ and $\mu(\epsilon=0.5)$

average degree grows, the overall scalability of anySCAN improves since the number of structural similarity calculations and the time for evaluating them both grow as well. On the other hand, when the average cluster coefficient is high, the overlap among the neighborhoods of vertices is also large, thus leading to more conflicts during Step 2 and 3 of anySCAN. This reduces the overall scalability. Figure 16 shows the scalability of anySCAN using multiple threads when varying the average degrees and cluster coefficients. Though there are some small fluctuations, the above trends are generally observed in most cases.

\subsection{Dynamic Anytime SCAN}

Since danySCAN is the first approach for weighted dynamic structural graph clustering, we compare it with dSCAN [8], an extension of pSCAN for dynamic graphs, also in its nonweighted mode with two operations including insertion and deletion. Moreover, since it processes updates in bulks, we compare it with pSCAN [5] and anySCAN [7] for fully reclustering the whole graphs. Unless otherwise stated, we use default parameters $\mu=5, \epsilon=0.5$, and $\alpha=\beta=8192$.

Performance comparison. Figure 17 shows the performance comparisons among danySCAN and competitors for GR01 to GR03 for different parameters $\epsilon(\mu=5)$ and $\mu(\epsilon=0.5)$ and 4000 updates (2000 delete and 2000 insert operations). Clearly, danySCAN is much more efficient (up to 35.2 times faster) than pSCAN and anySCAN since it only updates the cluster structures rather than starting from scratch. Compared to dSCAN, danySCAN runs much faster than dSCAN on GR01 and GR02 and is comparable to dSCAN on GR03. The reason is that dSCAN reclusters with each update, thus incurring costs for repeatedly traversing the graph, while danySCAN only needs to update clusters one time using its bulk processing scheme. Moreover, the denser the graphs, the better the performance of danySCAN compared to dSCAN due to the higher graph traversal costs. For example, dSCAN runs much slower (24.8 times) than danySCAN on GR01 $(\bar{d}=127)$ and is slightly slower $(1.5$ times) than danySCAN on GR02 ( $\bar{d}=14.2)$.

Figure 18 shows the cumulative runtimes of different methods when the number of updates changes from 2000 to 10000 . While the runtimes of pSCAN and anySCAN remains stable, those of danySCAN and dSCAN increase. However, the performance changes for dSCAN are much larger than for danySCAN. Again, this is because the bulk

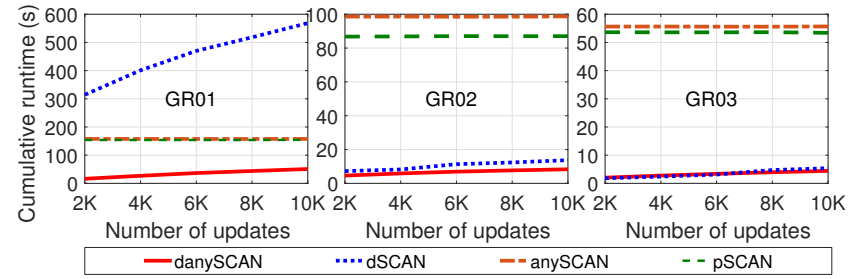

Fig. 18. Performance of danySCAN and others w.r.t. the number of updates for different datasets

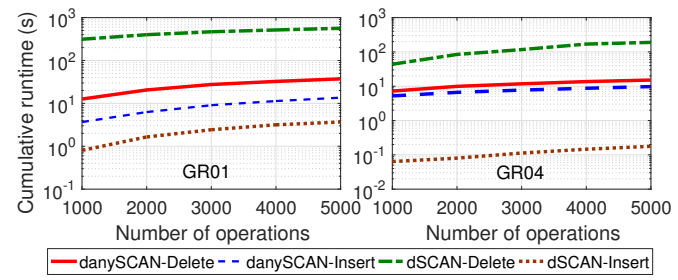

Fig. 19. Performance for different update operations
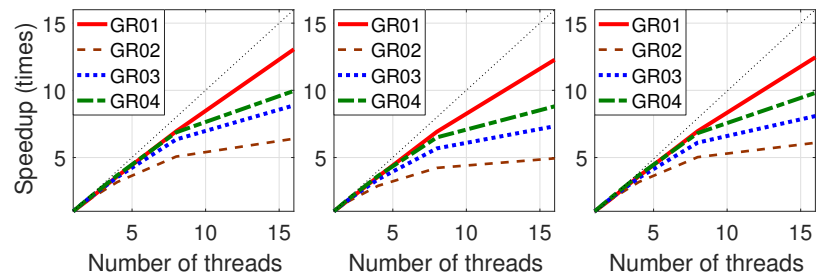

Fig. 20. Scalability of danySCAN w.r.t. the numbers of threads for different update operations including weight change (left), insertion (middle) and deletion (right), respectively

update scheme of danySCAN incurs less overhead than the batch update scheme of dSCAN.

Parameter analysis. As seen from Figure 17, when $\epsilon$ increases, the runtime of danySCAN decreases. The main reason is that with lower values of $\epsilon$, we have more core vertices. Thus, when there is a change in the graph, the number of affected clusters is higher. Thus, both dSCAN and danySCAN have to traverse a larger part of the graph for rebuilding clusters. Consequently, the overhead is higher, leading to performance degradation. Similarly, with lower values of $\mu$, we have more core vertices, leading to worse performance than with higher values of $\mu$.

Performance for different update operations. Figure 19 shows the cumulative runtimes of danySCAN and dSCAN for 1000 to 5000 insertion and deletion operations for GR01 and GR04. For both methods, deletion is more expensive than insertion. This is due to the fact that when an edge $(u, v)$ is deleted, it more likely will lead to a split of clusters. Thus, the algorithm must traverse the graph for rebuilding these clusters. In the insertion, it usually leads to a merge of clusters which is much cheaper due to the merging approach of both dSCAN and danySCAN. Moreover, while dSCAN processes the deletion cases much slower than danySCAN, it deals with the insertion ones very efficiently. For GR01 and 5000 insertion operations, dSCAN needs 3.67 seconds while danySCAN requires 13.58 seconds. However, with 5000 deletion operations, dSCAN consumes $564.85 \mathrm{sec}-$ onds compared to 37.30 seconds of danySCAN. danySCAN is designed to cope with mixed operations in its bulk update scheme and not optimized for each specific operation like dSCAN. However, since the deletion time is much larger than the insertion time, danySCAN still outperforms its 

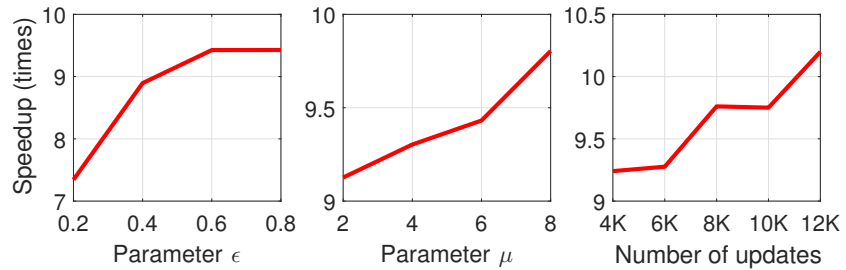

Fig. 21. Effect of parameters $\mu, \epsilon$ and the number of weight changes on the scalability of danySCAN on 16 threads for GR04 with 8000 updates

competitor if the number of insertions is not much larger than the number of deletions as demonstrated in Figure 17. For both types of operations, when the number of updates is larger, the better the performance of danySCAN is compared to that of dSCAN as observed from Figure 18 above. The weight change case of danySCAN has the performance between the insertion and the deletion cases since it naturally consists of these two.

\subsection{Dynamic Anytime SCAN on Multicore CPUs}

Scalability. Figure 20 shows the scalability of danySCAN w.r.t. the number of threads for different datasets and 10,000 different update operations using default parameters $\mu=$ $5, \epsilon=0.5$ and $\alpha=\beta=32768$. Overall, danySCAN scales very well with the number of threads. For example, using 16 threads, the speedup factor on GR01 is up to 13 times. Among different datasets, it has the worst performance on GR02. The reason has been examined in Section 5.2. GR02 is the sparsest graph with significantly varied vertex degrees, thus making workload balancing harder, which is reflected in the relatively weaker performance of danySCAN. The denser the graph, the better the scalability of danySCAN due to the larger overall workload for threads.

Weight change and deletion operations show better performance than insertion. The reason is simply that the more edges are deleted, the more clusters we need to rebuild and the heavier the update tasks which leads to better scalability.

Parameter analysis. As seen in Figure 21, smaller values of $\epsilon$ and $\mu$ will increase the number of core vertices, thus leading to workload imbalance and more super-node merges. This typically decreases the scalability as discussed in Section 5.2. When the number of updates is large, more vertices are affected in the bulk scheme. In turn, this increases the overall workload of threads. And consequently, it raises the scalability. The blocksize has similar effect on danySCAN compared to anySCAN like $\mu$ and $\epsilon$ (thus omitted here). The same results are observed for the other update cases.

\section{Related WORK AND Discussion}

Graph clustering techniques. Due to the ubiquitousness of graph like structures such as social networks, graph clustering techniques are becoming more and more important. There are graph clustering models such as modularity-based methods [1], graph partitioning [2], and structural graph clustering methods, which are our main focus here.

Structural graph clustering. Structural graph clustering, in particular the density-based approach of SCAN [3], is an active research topic with many extensions. For example, SCOT [25], HintClus [25], and gSkeletonClu [20] aim to solve the parameter setting problem of SCAN. DHSCAN
[26] and AHSCAN [27] are divisive and agglomerative hierarchical algorithms, respectively, using the structural similarity notion of SCAN. In this work, we focus on techniques that speed up the algorithm SCAN [3].

LinkScan* [28] improves the efficiency of SCAN using an edge sampling technique for reducing the number of similarity evaluations. However, it only approximates the result of SCAN unlike pSCAN [5] and SCAN++ [4].

pSCAN [5] is a recent state-of-the-art technique. Instead of calculating the full neighborhood of a vertice $p$, it only checks if $p$ is a core and then tries to connect $p$ to other core vertices from other clusters. This scheme significantly reduces structural evaluation and makes pSCAN one of the fastest variants of SCAN. The final cumulative runtimes of anySCAN are almost similar to those of pSCAN. However, anySCAN has the power of approximation and exact techniques at the same time in its anytime scheme.

SCAN++ [4] is the closest related work to anySCAN. It builds a set of pivots by performing neighborhood calculations for a vertex $p$, called a pivot, and expanding pivots for all nodes that are two-hop-away from $p$ in the same way as SCAN until it is converged. Then, it tries to connect pivots by examining and pruning bridge vertices that connect them. In this way, the number of similarity calculations is reduced. The goals of these steps bear some similarity with Step 1 and 2 of anySCAN, though anySCAN has a completely different angle. First, anySCAN randomly draws vertices for summarization and only keeps core vertices as super-nodes for further processing, thus limiting redundant similarity calculations since the number of super-nodes in anySCAN is much smaller than the number of pivots in SCAN++. Second, it connects super-nodes in a different manner as SCAN++ by examining two different kinds of connections (strong and weak ones) separately as well as processing only core vertices as super-nodes. Last, noise vertices are examined in the post processing step separately from the whole algorithm. Thus, it has better pruning power than SCAN++. And thus, it is more efficient.

Parallelizing SCAN. There exist some efforts for parallelizing SCAN. PSCAN [6] is a parallel version of SCAN using MapReduce for distributed computing. In [4], the authors also briefly introduce a MapReduce framework for SCAN++. The distributed model of MapReduce differs significantly from parallel computing in shared memory ones where memory is a contested resource, and latencies are small [29], [30]. Thus naively tranforming distributed algorithms to shared memory architectures will obviously be very inefficient as pointed out by many previous studies, e.g., [31]. To the best of our knowledge, anySCAN is the first parallel algorithm for SCAN on shared memory architectures such as multicore CPUs. Moreover, existing techniques such as pSCAN [5] and SCAN++ [4] incur many synchronizations that leave threads idle in a naive parallelization, which significantly reduces the scalability w.r.t. the number of threads. Recently, Takahashi et al. [9] introduce SCAN-XP, a parallel version of SCAN specifically designed for Intel Xeon Phi Coprocessors. This method, however, requires all structural similarities to be calculated. Thus, it is not a work-efficient parallel technique like anySCAN. It also is not designed to work with weighted graphs like anySCAN. 
Dynamic structural graph clustering. There exist some dynamic structural algorithms. DENGRAPH [19] is an incremental clustering algorithm designed to detect communities in large and dynamic social networks. Incorder [32] is an incremental version of gSkeletonClu [20]. Chang et al. [8] also introduces an incremental version of pSCAN, called dSCAN, which is closest to danySCAN. All these techniques update clusters in a batch mode with each insertion or deletion. For large number of updates, this scheme incurs many redundant calculations, thus decreasing their performance. On the other hand, danySCAN processes updates in a bulk mode, which is more efficient. Moreover, updates can be done in an anytime parallel way. In addition, danySCAN can handle dynamic weighted graphs.

\section{Conclusion}

In this paper, we propose an approach for accelerating the structural graph clustering algorithm SCAN. Our anytime algorithm, called anySCAN, quickly produces an approximate result in the beginning and continuously refines it for acquiring better results within arbitrary time constraints. This anytime scheme provides an efficient way for coping with large graphs. More interestingly, anySCAN is, at the same time, a parallel algorithm. Each iteration of its anytime scheme can be performed in parallel, thus further accelerating performance. To the best of our knowledge, anySCAN is the first anytime and parallel structural graph clustering algorithm. We additionally introduce an extension of anySCAN, called danySCAN, to parallelize processing of dynamic graphs. Experiments show that anySCAN has very good performance on large graph datasets in its anytime scheme. It also scales very well with the number of threads under shared memory architectures such as multicore CPUs.

In future work, we aim for a distribute extension of anySCAN to handle massive graph datasets on different machines, and applying our techniques to the study of patient data with sleep disorder symptoms.

\section{ACKNOWLEDGMENTS}

Special thank to Prof. Lijun Chang for providing us with the source code of pSCAN, Anh Le, Kenneth S. Bøgh, Panagiotis Bouros, and Prof. Hiroaki Shiokawa for their help and useful discussions. We also thank our anonymous reviewers for their fruitful comments. Part of this research was funded by a Villum postdoc fellowship and the CDP Life Project.

\section{REFERENCES}

[1] R. Guimer and L. A. N. Amaral, "Functional cartography of complex metabolic networks," Nature, 2005.

[2] J. Shi and J. Malik, "Normalized Cuts and Image Segmentation," IEEE Trans. Pattern Anal. Mach. Intell., vol. 22, no. 8, pp. 888-905, 2000.

[3] X. Xu, N. Yuruk, Z. Feng, and T. A. J. Schweiger, "SCAN: a structural clustering algorithm for networks," in $K D D, 2007$, pp. 824-833.

[4] H. Shiokawa, Y. Fujiwara, and M. Onizuka, "SCAN++: Efficient Algorithm for Finding Clusters, Hubs and Outliers on Large-scale Graphs," PVLDB, vol. 8, no. 11, pp. 1178-1189, 2015.

[5] L. Chang, W. Li, X. Lin, L. Qin, and W. Zhang, "pSCAN: Fast and Exact Structural Graph Clustering," in ICDE, 2016, pp. 481-490.

[6] W. Zhao, V. S. Martha, and X. Xu, "PSCAN: A Parallel Structural Clustering Algorithm for Big Networks in MapReduce," in AINA, 2013, pp. 862-869.
[7] S. T. Mai, M. S. Dieu, I. Assent, J. Jacobsen, J. Kristensen, and M. Birk, "Scalable and Interactive Graph Clustering Algorithm on Multicore CPUs," in ICDE, 2017, pp. 349-360.

[8] L. Chang, W. Li, L. Qin, W. Zhang, and S. Yang, "pSCAN: Fast and Exact Structural Graph Clustering," IEEE Trans. Knowl. Data Eng., vol. 29, no. 2, pp. 387-401, 2017.

[9] T. Takahashi, H. Shiokawa, and H. Kitagawa, "SCAN-XP: Parallel Structural Graph Clustering Algorithm on Intel Xeon Phi Coprocessors," in NDA@SIGMOD, 2017, pp. 6:1-6:7.

[10] S. Zilberstein, "Using Anytime Algorithms in Intelligent Systems," AI Magazine, vol. 17, no. 3, pp. 73-83, 1996.

[11] T. Kobayashi, M. Iwamura, T. Matsuda, and K. Kise, "An Anytime Algorithm for Camera-Based Character Recognition," in ICDAR, 2013, pp. 1140-1144.

[12] N. Begum, L. Ulanova, J. Wang, and E. J. Keogh, “Accelerating Dynamic Time Warping Clustering with a Novel Admissible Pruning Strategy," in KDD, 2015, pp. 49-58.

[13] S. T. Mai, I. Assent, and A. Le, "Anytime OPTICS: An Efficient Approach for Hierarchical Density-Based Clustering," in DASFAA, 2016, pp. 164-179.

[14] S. T. Mai, I. Assent, and M. Storgaard, "AnyDBC: An Efficient Anytime Density-based Clustering Algorithm for Very Large Complex Datasets," in KDD, 2016, pp. 1025-1034.

[15] S. T. Mai, X. He, J. Feng, and C. Böhm, "Efficient Anytime Densitybased Clustering," in SDM, 2013, pp. 112-120.

[16] S. T. Mai, X. He, J. Feng, C. Plant, and C. Böhm, "Anytime densitybased clustering of complex data," Knowl. Inf. Syst., vol. 45, no. 2 pp. 319-355, 2015.

[17] T. H. Cormen, C. E. Leiserson, R. L. Rivest, and C. Stein, Introduction to Algorithms (3. ed.). MIT Press, 2009.

[18] B. Chapman, G. Jost, and R. v. d. Pas, Using OpenMP: Portable Shared Memory Parallel Programming (Scientific and Engineering Computation). The MIT Press, 2007.

[19] T. Falkowski, A. Barth, and M. Spiliopoulou, "DENGRAPH: A Density-based Community Detection Algorithm," in Web Intelligence, 2007, pp. 112-115.

[20] H. Sun, J. Huang, J. Han, H. Deng, P. Zhao, and B. Feng, "gSkeletonClu: Density-Based Network Clustering via StructureConnected Tree Division or Agglomeration," in ICDM, 2010, pp. 481-490.

[21] J. Leskovec and A. Krevl, "SNAP Datasets: Stanford Large Network Dataset Collection," http://snap.stanford.edu/data, Jun. 2014.

[22] P. Boldi and S. Vigna, "The WebGraph framework I: Compression techniques," in WWW. Manhattan, USA: ACM Press, 2004, pp. 595-601.

[23] M. J. Zaki and W. M. Jr, Data Mining and Analysis: Fundamental Concepts and Algorithms. New York, NY, USA: Cambridge University Press, 2014.

[24] A. Lancichinetti, S. Fortunato, and F. Radicchi, "Benchmark graphs for testing community detection algorithms," Phys. Rev. E, vol. 78, p. 046110 , Oct 2008.

[25] D. Bortner and J. Han, "Progressive clustering of networks using Structure-Connected Order of Traversal," in ICDE, 2010, pp. 653656.

[26] N. Yuruk, M. Mete, X. Xu, and T. A. J. Schweiger, "A Divisive Hierarchical Structural Clustering Algorithm for Networks," in ICDM, 2007, pp. 441-448.

[27] — "AHSCAN: Agglomerative Hierarchical Structural Clustering Algorithm for Networks," in ASONAM, 2009, pp. 72-77.

[28] S. Lim, S. Ryu, S. Kwon, K. Jung, and J. Lee, "LinkSCAN*: Overlapping community detection using the link-space transformation," in ICDE, 2014, pp. 292-303.

[29] S. Tatikonda and S. Parthasarathy, "Mining Tree-Structured Data on Multicore Systems," PVLDB, vol. 2, no. 1, pp. 694-705, 2009.

[30] A. Ghoting, G. Buehrer, S. Parthasarathy, D. Kim, A. D. Nguyen, Y. Chen, and P. Dubey, "A Characterization of Data Mining Workloads on a Modern Processor," in DaMoN, 2005.

[31] M. M. A. Patwary, D. Palsetia, A. Agrawal, W. keng Liao, F. Manne, and A. N. Choudhary, "A new scalable parallel DBSCAN algorithm using the disjoint-set data structure," in SC, 2012, p. 62.

[32] H. Sun, J. Huang, X. Zhang, J. Liu, D. Wang, H. Liu, J. Zou, and Q. Song, "IncOrder: Incremental density-based community detection in dynamic networks," Knowl.-Based Syst., vol. 72, pp. $1-12,2014$. 


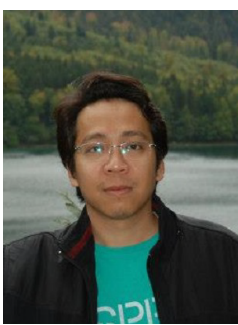

Son T. Mai is working at University of Grenoble Alpes, Grenoble, France. Before, he was at Aarhus University, Aarhus, Denmark and Ludwig-Maximilians-University of Munich (LMU), Munich, Germany.

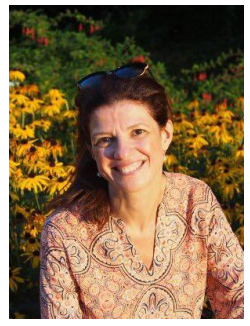

Sihem Amer-Yahia is a CNRS Research Director. Her interests are at the intersection of large-scale data management and social data exploration. Sihem held positions at QCRI, Yahoo! Research and AT\&T Labs. She served on the SIGMOD Executive Board, the VLDB Endowment, and the EDBT Board. She is Editorin-Chief of the VLDB Journal. Sihem serves as co-chair of PVLDB 2018, WWW 2018 Tutorials and ICDE 2019 Tutorials.

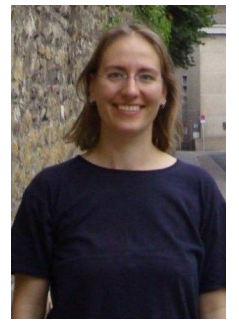

Ira Assent is an associate professor and head of the Data-Intensive Systems research group at the Department of Computer Science at Aarhus University, Denmark. Prior to joining Aarhus University in 2010, she was an assistant professor at Aalborg University, Denmark. Ira received her $\mathrm{Ph}$.D. in Computer Science with distinction from RWTH Aachen University, Germany in 2008.

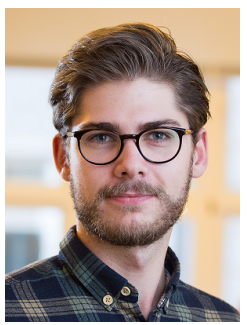

Mathias Skovgaard Birk received the MSc degree in computer science in 2017 from Aarhus University, Denmark, after finishing his thesis on incremental structural clustering of dynamic networks. His research interests include clustering, machine learning and design and development of distributed systems. He does not currently work in academia, but holds a position as software developer in a fintech company in Aarhus.

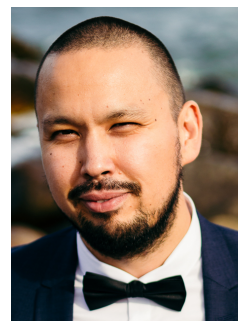

Martin Storgaard Dieu received the BSc degree in computer science at Southern University of Denmark in 2013 and his MSc degree in computer science at Aarhus University, Denmark in 2016. His main research interests are applied machine learning including graph clustering and anytime algorithms.

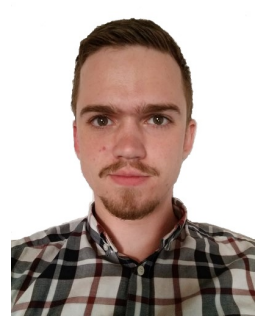

Jon Jacobsen received the BSc and MSc degree in computer science in 2014 and 2017 respectively from Aarhus University, Denmark. He studied as an exchange student at Nanyang Technological University's School of Computer Science and Engineering from July 2015 to December 2015. He is currently working as a software developer in Getinge Cetrea's R\&D department.

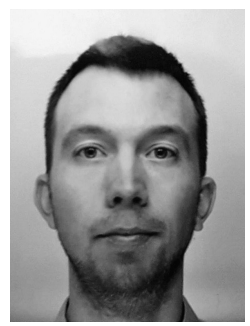

Jesper M. Kristensen received the MSc degree in computer science in 2016 from Aarhus University, Denmark. He is currently an IT consultant at Netcompany - IT and Business Consulting. 